\title{
TERRESTRIAL BIOTA CHECKLIST OF THE CHINIJO ARCHIPELAGO AND LOBOS (CANARY ISLANDS)
}

\author{
José María Fernández-Palacios*, Zaira Negrín, Silvia Fernández Lugo, \\ José Ramón Arévalo \& Lea de Nascimento
}

\begin{abstract}
A new checklist of the terrestrial biota from the easternmost islets from Canary Islands (Roque del Este, Roque del Oeste, Montaña Clara, Alegranza, La Graciosa and Lobos) isprovided as a result of a wide bibliographic and database compilation. The checklist includes bryophytes, vascular plants, fungi, chordates, arthropods and mollusks. Additionally, a short analysis of the fauna and flora species richness reveals that there are slightly more than 700 terrestrial species in the islets, the big majority of them being native, with $>25 \%$ endemics and $<10 \%$ exotics, what argues in favour of the high conservation quality of this protected area. By far, the more important taxa contributing to this biodiversity are arthropods and vascular plants, as happen in the rest of the archipelago. Up to seven different taxa, four spiders, one bird -already extinct-, one snail and one vascular plant, have been found to be endemic to the islets. Finally, the availability of this new checklist will contribute significantly towards more accurate biogeographic analyses of the Canarian biota, because until now the islets' biota distribution was not disaggregated of the main islands, Lanzarote and Fuerteventura, to which politically belong.
\end{abstract}

KEYWORDs: biogeographical analysis, compilation, species distribution, terrestrial biota.

\section{LISTADO DE LA BIOTA TERRESTRE DEL ARCHIPIÉLAGO CHINIJO Y LOBOS (ISLAS CANARIAS)}

\section{RESUMEN}

Se presenta un nuevo listado de la biota terrestre de las isletas más orientales del archipiélago canario (Roque del Este, Roque del Oeste, Montaña Clara, Alegranza, La Graciosa y Lobos) resultado de una exhaustiva recopilación bibliográfica y de bases de datos. El listado incluye briófitos, plantas vasculares, hongos, cordados, artrópodos y moluscos. Un breve análisis estadístico de la flora y fauna de los islotes revela que existen algo más de 700 especies en el conjunto de los mismos, la gran mayoría de ellas nativas, con más de un $25 \%$ de endemismos y menos de un $10 \%$ de especies exóticas, lo que avala la alta calidad de estos espacios protegidos. Con diferencia los taxa que más contribuyen a la biodiversidad inventariada son los artrópodos y las plantas vasculares, como ocurre en el resto del archipiélago. Hasta siete taxones diferentes, cuatro especies de arańa, un ave -ya extinta-, un molusco y una planta vascular, son endémicos de estas isletas. La disponibilidad de esta lista contribuirá a la realización de análisis biogeográficos más rigurosos, pues hasta ahora la biota de las isletas no se encontraba desagregada de las islas mayores, Lanzarote y Fuerteventura, a las que pertenecen políticamente.

PALABRAS ClAVE: análisis biogeográfico, compilación, distribución de especies, biota terrestre. 


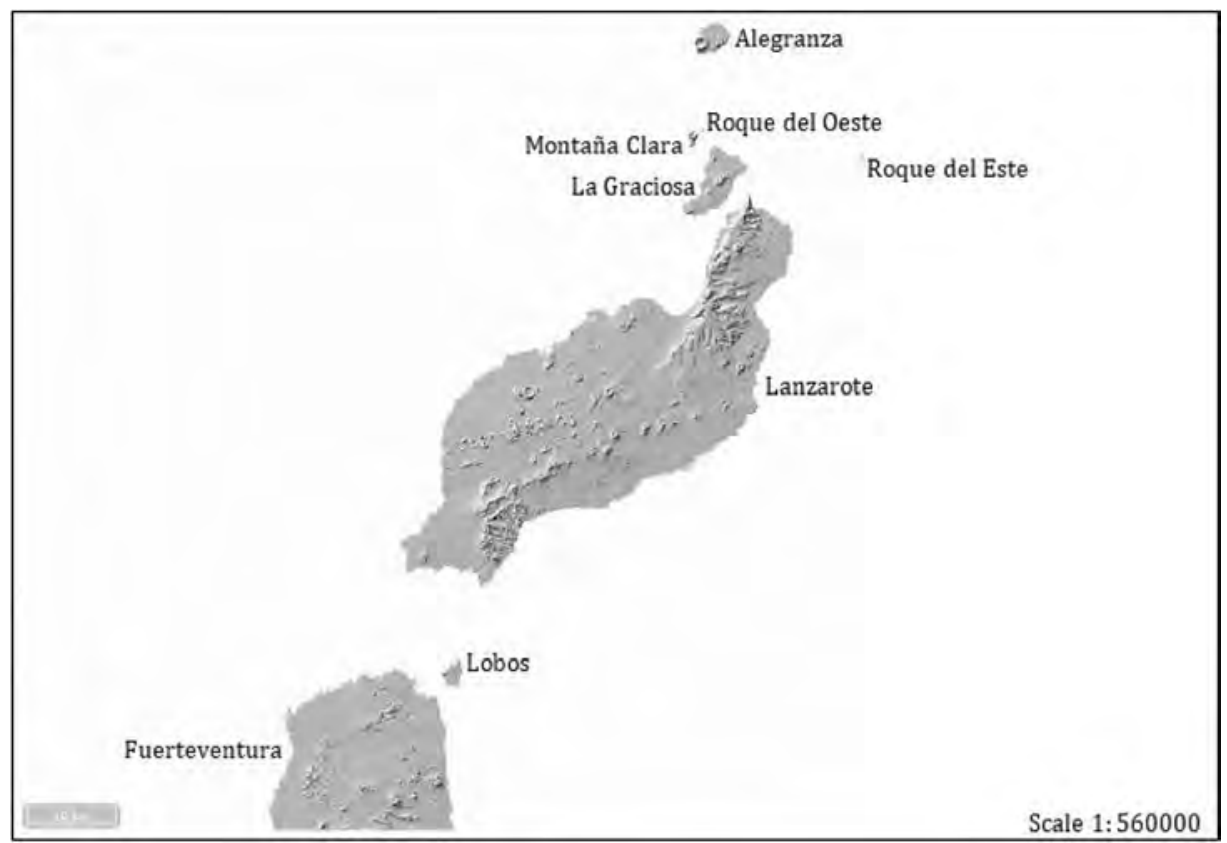

Figure 1. Geographical location of the islets considered in this study.

Despite the outstanding contribution that the existing species checklists of the NE Atlantic archipelagos (Arechavaleta et al. 2005, 2010; Borges et al. 2008, 2010) have provided for the recent progress of Macaronesian biogeography in particular and island biogeography in general (Emerson and Kolm, 2005; Whittaker et al. 2008; Rijsdijk et al. 2014; etc.), the Canarian checklist (Arechavaleta et al. 2010) is still counting with an important bias. This is that the species distribution information of the islets belonging to the so-called Archipiélago Chinijo (north to Lanzarote), comprising Roque del Este, Roque del Oeste, Montaña Clara, Alegranza and La Graciosa), as well as the information of Lobos islet, north to Fuerteventura (fig. 1), has been aggregated respectively within Lanzarote and Fuerteventura. Actually all these islets were, together their main respective islands, part of the Pleistocene island called Mahan, which extended in the Last Glacial Maximum (ca. 18 Ky BP) for more than $5000 \mathrm{~km}^{2}$ (Fernández-Palacios et al. 2011), but have became different insular entities at least ca. $15 \mathrm{Ky} \mathrm{BP,} \mathrm{during} \mathrm{the} \mathrm{last} \mathrm{deglaciation.}$

* Departamento de Botánica, Ecología y Fisiología Vegetal, Universidad de La Laguna, La Laguna, 38205, Tenerife, Spain. Corresponding autor: jmferpal@gmail.com. 
Contrastingly, other Macaronesian archipelagos' checklists, provide information about the islets species distribution to a large extent disaggregated from their respective main islands. For instance, Cape Verde checklist separates the information of the islets of Branco and Raso, close to Santa Luzia and São Nicolau, from these two islands, although this is not the case for Rombos islet, which is included within Brava (Arechavaleta et al. 2005). On the other hand, Madeira checklist disaggregates the information from Desertas and Salvajes archipelagos, although not of the islets included within them (Deserta Grande, Bugio and Ilheu Chao in Desertas and Selvagem Grande, Selvagem Pequena and Ilheu de Fora in Selvagens, respectively) (Borges et al. 2008). Finally, Azores disaggregates Corvo information from Flores (Borges et al. 2010), but there is no entrance for Formigas, a tiny islet halfway between Santa Maria and São Miguel. Remarkably, all those islets with disaggregated information are smaller than La Graciosa, and, with the exception of Corvo, than Alegranza or Lobos.

Some consequences of this lack of disaggregation for islets species distribution are, for instance, that the Canarian checklist includes islets endemic taxa attributed either to Fuerteventura or to Lanzarote. These errors cause loss of information and precision, generating inaccurate lists and thus, results and interpretations. Furthermore, counting with the islets' checklist biogeographic analyses could be performed with 11 (not including rocks) or even 13 (including the rocks) items and not only with seven, as use to happen today, where El Hierro $\left(270 \mathrm{~km}^{2}\right)$ is considered the smallest island. With the new checklist the island area range will be incremented in several orders of magnitude.

Thus, our aim in this study is to generate a new, updated checklist of the terrestrial biota from Chinijo's archipelago and Lobo's islet through a wide bibliographical and database compilation of all the terrestrial flora and fauna been described for these areas.

The checklist is based on published or unpublished information obtained from different sources either for their vascular flora (Kunkel, 1970, 1971; Marrero, 1991), fungi (León Arencibia et al. 1991; Beltrán-Tejera et al. 1998; Bordallo et al. 2012; Chávez-Barreto et al. 2016), vertebrate fauna (Martín and Lorenzo, 2001; Martín et al. 2003; Rodríguez et al. 2003; Lorenzo, 2007; Siverio et al. 2009; Ramírez et al. 2014), invertebrate fauna (Oromí and Arechavaleta, 1995; Pérez et al. 2003; Oromí et al. 2003; Macías et al. 2004), general approachs about specific islets, such as La Graciosa (González Viera et al. 1996), Montaña Clara (Wildpret et al. 1997; Varios autores, 2006) or Lobos (Romero, 2017), and finally personal communications (such as Prof. González Mancebo for bryophytes or Prof. Oromí for invertebrates). Although Kunkel $(1970,1971)$ included cultivated (garden, agriculture, plantations) species, either growing wild or not, in this checklist cultivated species growing wild have been included as exotics, while those not growing wild have been excluded.

Species have been listed according to their presence (+), absence (-) or data inexistence $(*)$ in the different islets analysed: Roque del Este (RE), Roque del Oeste (RO), Montańa Clara (MC), Alegranza (AL), La Graciosa (LG) and Lobos (LO). Following the Canarian checklist procedure (Arechavaleta et al. 2010), for each 
taxon its origin (Or) [secure native (NS), likely native (NP), possible native (NO), likely introduced (IP), non-invasive secure introduced (IS) and invasive introduced (II)] and endemicity status (En) [endemics subspecies (ESS), endemic species (ES), endemics genus (EG) or islet endemic (IE)] are given. Information about extirpated or extinguished taxa (e) or with doubtful presence (?) is also included. When for a specific species only doubtful presences are referred along the study area, the species has been omitted from the list. By contrast, if there is at least one confirmed presence $(+)$ along the study area, doubtful presences are considered. Species and the families to whom they belong have been listed according to alphabetic order within taxonomic phyla.

As was to be expected, the survey effort has not been the same all over the islets studied, so that for instance, vascular plants have been especially well studied for Lobos, and the arthropod fauna for Montaña Clara. The two larger islets (La Graciosa and Alegranza) have received intermediate sampling efforts and finally, due to their inaccessibility the sampling effort for the tiny rocks has been very limited. Thus, the species list is simultaneously a trade off of the islets true diversity on the on hand, and on the inventory effort on the other, as seems to happen universally (Hortal et al. 2007). Even so, we consider the resulting checklist (see appendix) as a first step towards the disaggregation of the islets dataset and thus a real basis for biogeographic analyses improvement.

The checklist includes six taxonomic groups: bryophytes, vascular plants, arthropods, mollusks, chordates and fungi. Unfortunately, we have not been able to find sources for lichen distributions in those islets, although it is known that there exist some (Varios autores, 2006).

On the other hand, aiming completeness, the checklist has included species that have been extinct (such as the malpaís mouse Malpaisomys insularis, the black oystercatcher Haematopus meadewaldoi or the endemic Chinijo archipelago stonechat Saxicola dacotiae ssp. murielae) or extirpated (such as the Egyptian vulture Neophron percnopterus or the osprey Pandion haliaetus) in historical times from all or some of the islets.

For the statistical analysis we have consider extinct or extirpated taxa ( $e$ in the checklist, see appendix) as species presence $(+)$, whereas doubtful presence (? in the checklist) or no data ${ }^{*}$ in the checklist) was precautionary consider as species absence.

704 different terrestrial species have been found growing on the islets (see appendix), although we believe that there should be more because the impossibility of finding lichen and other invertebrate information further than arthropods or mollusks species. Leaving apart the two very tiny rocks, Roques del Este y del Oeste, with 33 and 16 species respectively, the species richness of the different islets is remarkably similar, around 300 species, despite their very different area, varying from $1.33 \mathrm{~km}^{2}$ (Montaña Clara) to $27.3 \mathrm{~km}^{2}$ (La Graciosa) (table 1). Within the species present, from a chorological perspective the non-endemic native element is by far the more abundant (varying from 75 to $49 \%$ among the different islets), followed by the endemic species (shifting from 45 to $25 \%$ ) and finally, by the introduced species, always $<10 \%$ and almost absent of the rocks. The very low contribution of 
the exotic species all over the studied phyla on the islet diversity argues in favour of the high conservation status of this natural area (table 2).

\begin{tabular}{|c|c|c|c|c|c|}
\hline \multicolumn{6}{|c|}{ TABLE 1. GEOGRAPHICAL DATA OF THE ISLETS STUDIED } \\
\hline IsLET & $\operatorname{ArEA}\left(\mathrm{km}^{2}\right)$ & Altitude (m) & $\begin{array}{l}\text { Closest main } \\
\text { ISLAND }\end{array}$ & $\begin{array}{l}\text { DistanCE TO THE } \\
\text { MAIN ISLAND }(\mathrm{km})\end{array}$ & $\begin{array}{l}2017 \text { HUMAN } \\
\text { POPULATION }\end{array}$ \\
\hline La Graciosa & 27.3 & 266 & Lanzarote & 1.15 & 721 \\
\hline Alegranza & 10.2 & 289 & Lanzarote & 16.56 & - \\
\hline Montaña Clara & 1.33 & 256 & Lanzarote & 8.39 & - \\
\hline Roque del Este & 0.06 & 86 & Lanzarote & 10.96 & - \\
\hline Roque del Oeste & 0.01 & 43 & Lanzarote & 9.79 & - \\
\hline Isla de Lobos & 4.58 & 122 & Fuerteventura & 1.95 & 4 \\
\hline
\end{tabular}

\begin{tabular}{|c|c|c|c|c|c|c|c|}
\hline \multicolumn{8}{|c|}{$\begin{array}{l}\text { TABLE 2. NUMBER OF SPECIES AND PERCENTAGE OVER THE TOTAL ISLET RICHNESS } \\
\text { IN BRACKETS, ACCORDING TO ITS BIOGEOGRAPHICAL ORIGIN }\end{array}$} \\
\hline $\begin{array}{l}\text { ChOROLOGICAL } \\
\text { STATUS }\end{array}$ & $\begin{array}{l}\text { ROQUe } \\
\text { DEL EsTE }\end{array}$ & $\begin{array}{l}\text { RoQue DEL } \\
\text { OESTE }\end{array}$ & $\begin{array}{l}\text { Montaña } \\
\text { Clara }\end{array}$ & Alegranza & La Graciosa & $\begin{array}{l}\text { ISLA DE } \\
\text { LOBOS }\end{array}$ & TотAL \\
\hline Endemic sp. & $\begin{array}{l}15 \\
(45.4 \%)\end{array}$ & $\begin{array}{c}4 \\
(25 \%)\end{array}$ & $\begin{array}{l}96 \\
(31.9 \%)\end{array}$ & $\begin{array}{c}81 \\
(26.4 \%)\end{array}$ & $\begin{array}{c}84 \\
(26.9 \%)\end{array}$ & $\begin{array}{c}74 \\
(24.7 \%)\end{array}$ & $\begin{array}{c}222 \\
(31.4 \%)\end{array}$ \\
\hline $\begin{array}{l}\text { Non-endemic } \\
\text { native sp. }\end{array}$ & $\begin{array}{c}16 \\
(48.5 \%)\end{array}$ & $\begin{array}{c}12 \\
(75 \%)\end{array}$ & $\begin{array}{c}194 \\
(64.5 \%)\end{array}$ & $\begin{array}{c}212 \\
(69.1 \%)\end{array}$ & $\begin{array}{c}198 \\
(64.3 \%)\end{array}$ & $\begin{array}{c}194 \\
(65.8 \%)\end{array}$ & $\begin{array}{c}418 \\
(59.5 \%)\end{array}$ \\
\hline Exotic sp. & $\begin{array}{c}2 \\
(6.1 \%)\end{array}$ & $\begin{array}{c}0 \\
(-)\end{array}$ & $\begin{array}{c}11 \\
(3.6 \%)\end{array}$ & $\begin{array}{c}14 \\
(4.6 \%)\end{array}$ & $\begin{array}{c}27 \\
(8.8 \%)\end{array}$ & $\begin{array}{c}28 \\
(9.5 \%)\end{array}$ & $\begin{array}{c}64 \\
(9.1 \%)\end{array}$ \\
\hline Total sp. & 33 & 16 & 301 & 307 & 309 & 296 & 704 \\
\hline
\end{tabular}

In respect to the contribution of the different taxonomic groups to the island biodiversity, arthropods and vascular plants are the more abundant groups with > $90 \%$ of the species present, whereas the rest only account for an $8 \%$ of the species richness (table 3). Interestingly there are two exclusive taxa shared by Montaña Clara and Alegranza, the (already extinct) passerine landbird (Saxicola dacotiae ssp. murielae) and the spider Salticus alegranzaensis; four more are exclusive from Alegranza, the spiders Cerbalus alegranzaensis, Dysdera alegranzaensis and Eurypoena tuberosa ssp. alegranzaensis, and the snail Cryptella alegranzae; and finally, one vascular plant, Limonium ovalifolium ssp. canariensis, exists exclusively in Lobos. No exclusive species has been reported for La Graciosa, neither for the Roques so far.

Summarizing we present here a new biogeographical tool, that even needing some improvement and refinements as the incorporation of new taxonomic groups (such as the lichens), constitutes a significant step towards counting with a species checklist for the complete Canarian archipelago. 


\begin{tabular}{|c|c|c|c|c|c|c|c|}
\hline \multicolumn{8}{|c|}{$\begin{array}{l}\text { TABLE 3. SPECIES DISTRIBUTION AND PERCENTAGE OVER THE TOTAL ISLET BIOTA } \\
\text { IN BRACKETS OF THE DIFFERENT TAXONOMIC GROUPS PER ISLET }\end{array}$} \\
\hline TAXA & $\begin{array}{l}\text { ROQUE } \\
\text { DEL ESTE }\end{array}$ & $\begin{array}{l}\text { ROQUE DEL } \\
\text { OESTE }\end{array}$ & $\begin{array}{l}\text { Montaña } \\
\text { Clara }\end{array}$ & Alegranza & La Graciosa & $\begin{array}{l}\text { ISLA DE } \\
\text { LOBOS }\end{array}$ & Total \\
\hline Arthropods & $\begin{array}{c}13 \\
(39.4 \%)\end{array}$ & * & $\begin{array}{c}171 \\
(56.8 \%)\end{array}$ & $\begin{array}{c}142 \\
(46.2 \%)\end{array}$ & $\begin{array}{c}110 \\
(35.7 \%)\end{array}$ & $\begin{array}{c}96 \\
(32.5 \%)\end{array}$ & $\begin{array}{c}390 \\
(55.5 \%)\end{array}$ \\
\hline Mollusks & $\begin{array}{c}1 \\
(3 \%)\end{array}$ & * & $\begin{array}{c}6 \\
(2.0 \%)\end{array}$ & $\begin{array}{c}5 \\
(1.6 \%)\end{array}$ & $\begin{array}{c}1 \\
(0.3 \%)\end{array}$ & $\begin{array}{c}3 \\
(1 \%)\end{array}$ & $\begin{array}{c}10 \\
(1.4 \%)\end{array}$ \\
\hline Birds & $\begin{array}{c}7 \\
(21.2 \%)\end{array}$ & $\begin{array}{c}8 \\
(50 \%)\end{array}$ & $\begin{array}{c}19 \\
(6.3 \%)\end{array}$ & $\begin{array}{c}24 \\
(7.8 \%)\end{array}$ & $\begin{array}{c}22 \\
(7.1 \%)\end{array}$ & $\begin{array}{c}22 \\
(7.4 \%)\end{array}$ & $\begin{array}{c}33 \\
(4.7 \%)\end{array}$ \\
\hline Reptiles & $\begin{array}{c}2 \\
(6 \%)\end{array}$ & $\begin{array}{c}2 \\
(12.5 \%)\end{array}$ & $\begin{array}{c}2 \\
(0.6 \%)\end{array}$ & $\begin{array}{c}2 \\
(0.6 \%)\end{array}$ & $\begin{array}{c}2 \\
(0.7 \%)\end{array}$ & $\begin{array}{c}3 \\
(1 \%)\end{array}$ & $\begin{array}{c}3 \\
(0.4 \%)\end{array}$ \\
\hline Mammals & $\begin{array}{c}0 \\
(-)\end{array}$ & $\begin{array}{c}0 \\
(-)\end{array}$ & $\begin{array}{c}1 \\
(0.3 \%)\end{array}$ & $\begin{array}{c}2 \\
(0.6 \%)\end{array}$ & $\begin{array}{c}4 \\
(1.4 \%)\end{array}$ & $\begin{array}{c}4 \\
(1.4 \%)\end{array}$ & $\begin{array}{c}5 \\
(0.6 \%)\end{array}$ \\
\hline Bryophytes & $\begin{array}{c}0 \\
(-)\end{array}$ & $\begin{array}{c}0 \\
(-)\end{array}$ & $\begin{array}{c}2 \\
(0.6 \%)\end{array}$ & $\begin{array}{c}6 \\
(1.8 \%)\end{array}$ & $\begin{array}{c}4 \\
(1.3 \%)\end{array}$ & $\begin{array}{c}7 \\
(2,3 \%)\end{array}$ & $\begin{array}{c}11 \\
(1.6 \%)\end{array}$ \\
\hline Fungi & $\begin{array}{c}0 \\
(-)\end{array}$ & $\begin{array}{c}0 \\
(-)\end{array}$ & $\begin{array}{c}0 \\
(-)\end{array}$ & $\begin{array}{c}2 \\
(0.6 \%)\end{array}$ & $\begin{array}{c}4 \\
(1.3 \%)\end{array}$ & $\begin{array}{c}3 \\
(1 \%)\end{array}$ & $\begin{array}{c}8 \\
(1.1 \%)\end{array}$ \\
\hline $\begin{array}{l}\text { Vascular } \\
\text { plants }\end{array}$ & $\begin{array}{c}10 \\
(30.3 \%)\end{array}$ & $\begin{array}{c}6 \\
(37.5 \%)\end{array}$ & $\begin{array}{c}100 \\
(33.2 \%)\end{array}$ & $\begin{array}{c}124 \\
(40.4 \%)\end{array}$ & $\begin{array}{c}162 \\
(52.6 \%)\end{array}$ & $\begin{array}{c}158 \\
(53.6 \%)\end{array}$ & $\begin{array}{c}244 \\
(34.7 \%)\end{array}$ \\
\hline Total & 33 & 16 & 301 & 307 & 309 & 296 & 704 \\
\hline
\end{tabular}

RECIBIDO: octubre de 2017, ACEPTADO: noviembre de 2018

\section{ACKNOWLEDGMENTS}

The authors are very gratefull with our Departament colleagues Proffesors Juana María González Mancebo and Esperanza Beltrán Tejera for facilitating information concerning the distribution of bryophytes and fungi. Prof. Dr. Pedro Oromí (Zoology Department) provided us unpublished data of invertebrate distribution in the islets. Finally, two anonimous referees contributed to the improvement if this manuscript.

\section{AUTHORS' CONTRIBUTION}

Conceptualization: JMFP.

Methodology and field work: JMFP, ZN, SFL, JRA, LdN.

Data analysis: JMFP.

Original draft: ZN, JMFP.

Review and edition of the final draft: all authors. 


\section{REFERENCES}

Arechavaleta, M., Zurita, N., Marrero, M.C. and Martín, J.L. (eds.) 2005. Lista preliminar de especies silvestres de Cabo Verde (hongos, plantas y animales trerrestres). 2005. Gobierno de Canarias. 155 pp.

Arechavaleta, M., Rodríguez, S., Zurita, N. and García, A. (eds.) 2010. Lista de especies silvestres de Canarias. Hongos, plantas y animales terrestres. 2009. Gobierno de Canarias. 579 pp.

Beltrán-Tejera, E., Bañares-Baudet, A. and Rodríguez-Armas, J.L. 1998. «Gasteromycetes of the Canary islands. Some notewhorty records». Mycotaxon, 67: 439-453.

Bordallo, J.J., Rodríguez, A., Honrubia, M. and Morte, A. (2012) Terfezia canariensis sp. nov., una nueva especie de trufa encontrada en las Islas Canarias. Cantarela, 56: 1-8.

Borges, P.A.V., Abreu, C., Aguilar, A.M.F., Carvalho, P., Jardim, R., Melo, I., Oliveira, P., Sérgio, C., Serrano, A.R.M. and Vieira, P. (eds.) 2008. A list of the terrestrial fungi, flora and fauna of Madeira and Selvagens archipelagos. Direcção Regional do Ambiente da Madeira and Universidade dos Açores. 440 pp.

Borges, P.A.V., Costa, A., Cunha, R., Gabriel, R., Gonçalves, V., Martins, A.F., Melo, I., Parente, M., Raposeiro, P., Rodrigues, P., Santos, R.S., Silva, L., Vieria, P. and Vieria, V. (eds.) 2010. A list of the terrestrial and marine biota from the Azores. Principia, Cascais, 432 pp.

Chávez Barreto, D., Negrín Piñero, R., Govantes Moreno, F., Torres Cabrera, J.M., Rodríguez Cabrera, C.C., Clacines Martín, L., Lantigua Calderín, J.L., Naranjo Rodríguez, R. López Quintanilla, J.F., Suárez Gil, A., Velaz Vergara, J.I. and Escobio García, V.J. 2016. «Adiciones a la biota fúngica de las Islas Canarias». Cantarela, 72: 1-4.

Emerson, B.C. and Kolm, N. 2005. Species diversity can drive speciation. Nature 434, 1015-1017.

Fernández-Palacios, J.M., de Nascimento, L., Otto R., Delgado, J., García-del-Rey, E., Arévalo, J.R. and Whittaker, R.J. 2011. «A reconstruction of Palaeo-Macaronesia, with particular reference to the long-term biogeography of the Atlantic island laurel forests». Journal of Biogeography 38: 226-246.

González Viera, F.J., Morín Pérez, P. and Acosta Rodríguez, J.E. 1996. La Graciosa: estudio histórico y geográfico. Centro de la Cultura Popular Canaria.

Hortal, J.M., Lobo, J.M. and JimÉnez-VAlverde, A. 2007. «Limitations of Biodiversity Databases: Case Study on Seed-Plant Diversity in Tenerife, Canary Islands». Conservation Biology, 21: 853-863.

Kunkel, G. 1970. Flórula de la isla de Lobos (Islas Canarias). Monogr. Biol. Can., 1: 1-60.

Kunkel, G. 1971. «La Vegetación de La Graciosa y notas sobre Alegranza, Montaña Clara y el Roque del Infierno (Islas Canarias)». Monogr. Biol. Can., 2: 1-67.

León Arencibia, M.C., Beltrán-Tejera, E. and Wildpret, W. 1991. "Contribución al estudio de la flora micológica de las Canarias orientales. La Graciosa», in Homenaje al Prof. Dr. T. Bravo. Servicio de Publicaciones de la Universidad de La Laguna. I: 452-471.

Lorenzo, J.A. (ed.) 2007. Atlas de las aves nidificantes en el archipiélago canario (1997-2003) Dirección General de Conservación de la Naturaleza-Sociedad Española de Ornitología, Madrid. 520 pp. 
Macías, N., Pérez, A.J., López, H. and Oromí, P. 2004. Fauna de artrópodos de Montaña Clara (Islas Canarias) III: arácnidos, miriápodos y crustáceos terrestres. Rev. Acad. Canar. Cienc., XV (No. 3-4): 53-68.

Marrero, A. 1991. «La flora y la vegetación del Parque Natural de "Los islotes del norte de Lanzarote y Riscos de Famara”. Su situación actual». In: Dias, E., Carretas, J.P. and Cordeiro, P. (eds.) Primeras Jornadas Atlânticas de Protecção do Meio Ambiente. Angra do Heroismo. pp. 195-214.

Martín A. and Lorenzo, J.A. 2001. Aves del Archipiélago Canario. Francisco Lemus Editor. La Laguna, $774 \mathrm{pp}$.

Martín, A., Alonso, J. and Rodríguez, B. 2003. «Los islotes del norte de Lanzarote. Una propuesta de Parque Nacional». El Indiferente, 14: 16-25.

Oromí P., López, H., Arechavaleta, M., Contreras-Díaz, H. and Rodríguez, B. 2003. «The arthropod fauna of Montaña Clara (Canary Islands) I: Coleoptera». Vieraea, 31: 167-182.

Oromí, P. and Arechavaleta, M. 1995. Fauna de invertebrados del Parque Natural del Archipiélago Chinijo. Viceconsejería Medio Ambiente (unpublished report). pp. 3-10.

Pérez, A.J., Morales, E., Oromí, P. and López, H. 2003. «Arthropod fauna of Montaña Clara (Canary Islands) II: Hexapoda (except Coleoptera)». Vieraea, 31: 237-251.

Rijsdijk, K.F., Hengl, T., Norder, S.J., Otto, R., Emerson, B.C., Ávila, S., López, H., van Loon, E., TJørve, E. and Fernandez-Palacios, J.M. 2014. «Quantifying surface-area changes of volcanic islands driven by Pleistocene sea-level cycles: biogeographical implications for the Macaronesian archipelagos». J. Biogeogr., 41: 1242-1254.

Ramírez, J., Roldán, J. and Moreno, W. 2014. «El guirre recupera lentamente sus territorios en las Canarias orientales». Quercus, 346: 32-37.

Rodríguez, B., De León, L., Martín, A., Rodríguez, B. and Nogales, M. 2003. «Status and distribution of breeding seabirds in the Northern islets of Lanzarote». Atlantic Seabirds, 5: 41-56.

Romero, I. 2017. Isla de Lobos, Naturaleza e historia. Ediciones Remotas, 135 pp.

Siverio, M., Rodríguez, B. and Siverio, F. 2009. «Halcón tagarote Falco peregrinus pelegrinoides en las islas Canarias", en del Moral, J.C. (ed.). El halcón peregrino en España. Sociedad Española de Ornitología, Madrid. 52-58 pp.

Varios autores 2006. Documento informativo del Plan director de la Reserva Natural Integral de los islotes. Consejería de Medio ambiente y ordenación territorial, Gobierno de Canarias.

Whittaker, R.J., Triantis, K.T. and Ladle, R. 2008. "A general dynamic theory of oceanic island biogeography». J. Biogeogr., 35: 977-994.

Wildpret, W., Beltrán Tejera, W.E. and León Arencibia, M.C. 1997. «Flora and vascular vegetation of the islet of Montaña Clara (Canary Islands)»: 237-246. Islands and High Mountain Vegetation: Biodiversity, Bioclimate and Conservation. IAVS Symposium Tenerife (April 1993). Proceeding Book. Universidad de La Laguna, serie Informes, n. ${ }^{\circ} 40$. 


\section{APPENDIX: SPECIES CHECKLIST}

\begin{tabular}{|c|c|c|c|c|c|c|c|c|}
\hline PHYLUM CHORDATA & 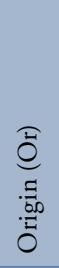 & 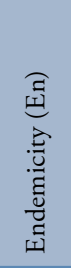 & 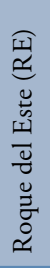 & 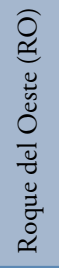 & 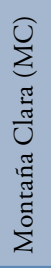 & 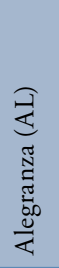 & 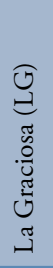 & 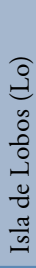 \\
\hline \multicolumn{9}{|l|}{ Class Aves } \\
\hline \multicolumn{9}{|l|}{ Accipitridae } \\
\hline Neophron percnopterus L., 1758 & NS & & - & - & e & + & - & e \\
\hline \multicolumn{9}{|l|}{ Alaudidae } \\
\hline $\begin{array}{l}\text { Calandrella rufescens ssp. rufescens Vieillot, } \\
1820\end{array}$ & NS & ESS & - & - & - & - & + & + \\
\hline \multicolumn{9}{|l|}{ Apodidae } \\
\hline Apus pallidus ssp. brehmorum Shelley, 1870 & NS & & - & - & - & - & + & - \\
\hline \multicolumn{9}{|l|}{ Burhinidae } \\
\hline Burhinus oedicnemus ssp. insularum Sassi, 1908 & NS & ESS & - & - & - & + & + & + \\
\hline \multicolumn{9}{|l|}{ Charadriidae } \\
\hline Charadrius alexandrinus L., 1758 & NS & & - & - & - & - & + & + \\
\hline \multicolumn{9}{|l|}{ Columbidae } \\
\hline Columba livia Gmelin, 1789 & NS & & $?$ & - & + & + & + & + \\
\hline \multicolumn{9}{|l|}{ Corvidae } \\
\hline $\begin{array}{l}\text { Corvus corax ssp. canariensis Hartert and } \\
\text { Kleinschmidt, } 1901\end{array}$ & NS & ESS & - & - & + & + & + & + \\
\hline \multicolumn{9}{|l|}{ Falconidae } \\
\hline Falco eleonorae Gené, 1839 & NS & & + & + & + & + & - & - \\
\hline Falco pelegrinoides Temminck, 1829 & NS & & + & - & + & + & - & + \\
\hline Falco tinnunculus ssp. dacotiae Hartert, 1913 & NS & ESS & - & - & + & + & + & + \\
\hline \multicolumn{9}{|l|}{ Fringillidae } \\
\hline $\begin{array}{l}\text { Bucanetes githagineus ssp. amantum Hartetrt, } \\
1903\end{array}$ & NS & ESS & - & - & - & + & + & + \\
\hline $\begin{array}{l}\text { Carduelis cannabina ssp. harterti Bannerman, } \\
1913\end{array}$ & NS & ESS & - & - & - & - & + & + \\
\hline \multicolumn{9}{|l|}{ Glareolidae } \\
\hline Cursorius cursor Latham, 1787 & NS & & - & - & - & - & $?$ & - \\
\hline \multicolumn{9}{|l|}{ Haematopodidae } \\
\hline Haematopus meadewaldoi Bannerman, 1913 & NS & ES & - & e & e & e & e & - \\
\hline \multicolumn{9}{|l|}{ Hydrobatidae } \\
\hline Hydrobates pelagicus ssp. pelagicus L., 1758 & NS & & + & + & + & + & + & + \\
\hline
\end{tabular}




\begin{tabular}{|c|c|c|c|c|c|c|c|c|}
\hline Oceanodroma castro Harcourt, 1851 & NS & & + & + & + & + & $?$ & + \\
\hline $\begin{array}{l}\text { Pelagodroma marina ssp. hypoleuca Webb, } \\
\text { Berthelot and Moquin-Tandon, } 1842\end{array}$ & NS & & - & - & + & + & ? & ? \\
\hline \multicolumn{9}{|l|}{ Laniidae } \\
\hline Lanius meridionalis ssp. koenigi Hartert, 1901 & NS & ESS & - & - & + & + & + & + \\
\hline \multicolumn{9}{|l|}{ Laridae } \\
\hline Larus fuscus $\mathrm{L}$. & NS & & - & - & + & + & - & - \\
\hline Larus michahellis ssp. atlantis Dwight, 1922 & NS & & + & $\mathrm{e}$ & + & + & + & + \\
\hline \multicolumn{9}{|l|}{ Motacillidae } \\
\hline Anthus berthelottii ssp. berthelotii Bolle, 1862 & NS & & - & - & + & + & + & + \\
\hline \multicolumn{9}{|l|}{ Otididae } \\
\hline $\begin{array}{l}\text { Chlamydotis undulata ssp. fuertaventurae Roth- } \\
\text { schild and Hartert, } 1894\end{array}$ & NS & ESS & - & - & - & - & + & - \\
\hline \multicolumn{9}{|l|}{ Pandionidae } \\
\hline Pandion haliaetus ssp. haliaetus L., 1758 & NS & & + & $\mathrm{e}$ & + & + & $\mathrm{e}$ & ? \\
\hline \multicolumn{9}{|l|}{ Phasianidae } \\
\hline Alectoris barbara ssp. koenigi Reich. 1899 & IS & & - & - & - & $\mathrm{e}$ & + & + \\
\hline \multicolumn{9}{|l|}{ Procellariidae } \\
\hline Bulweria bulwerii Jardine and Selby, 1828 & NS & & - & + & + & + & + & + \\
\hline Calonectris diomedea ssp. borealis Cory, 1881 & NS & & + & + & + & + & + & + \\
\hline Puffinus assimilis ssp. baroli Bonaparte, 1857 & NS & & - & - & + & + & ? & ? \\
\hline \multicolumn{9}{|l|}{ Recurvirostridae } \\
\hline Himantopus himantopus L., 1758 & NS & & & & & & & + \\
\hline \multicolumn{9}{|l|}{ Sternidae } \\
\hline Sterna hirundo ssp. hirundo L., 1758 & NS & & - & - & - & - & - & + \\
\hline \multicolumn{9}{|l|}{ Sylviidae } \\
\hline Sylvia conspicillata ssp. orbitalis Wahlberg, 1854 & NS & & - & - & $?$ & + & + & + \\
\hline \multicolumn{9}{|l|}{ Turdidae } \\
\hline Saxicola dacotiae ssp murielae Bannerman, 1913 & NS & IE & - & - & $\mathrm{e}$ & $\mathrm{e}$ & - & - \\
\hline \multicolumn{9}{|l|}{ Tytonidae } \\
\hline Tyto alba ssp. gracilirostris Hartert, 1905 & NS & ESS & - & - & ? & + & + & + \\
\hline \multicolumn{9}{|l|}{ Upupidae } \\
\hline Upupa epops L., 1758 & NS & & - & - & - & - & + & ? \\
\hline \multicolumn{9}{|l|}{ Class Reptilia } \\
\hline \multicolumn{9}{|l|}{ Gekkonidae } \\
\hline Tarentola angustimentalis Steindachner, 1891 & NS & ES & + & + & + & + & + & + \\
\hline \multicolumn{9}{|l|}{ Lacertidae } \\
\hline Gallotia atlantica Peters and Doria, 1882 & NS & ES & + & + & + & + & + & + \\
\hline
\end{tabular}




\begin{tabular}{|c|c|c|c|c|c|c|c|c|}
\hline \multicolumn{9}{|l|}{ Scincidae } \\
\hline Chalcides simonyi Steindachner, 1891 & NS & ES & - & - & - & - & - & + \\
\hline \multicolumn{9}{|l|}{ Class Mammalia } \\
\hline \multicolumn{9}{|l|}{ Felidae } \\
\hline Felis silvestris catus L., 1758 & II & & - & - & - & - & + & + \\
\hline \multicolumn{9}{|l|}{ Leporidae } \\
\hline Oryctolagus cuniculus L., 1758 & II & & - & - & - & + & + & + \\
\hline \multicolumn{9}{|l|}{ Muridae } \\
\hline $\begin{array}{l}\text { Malpaisomys insularis Hutterer, López- } \\
\text { Martínez and Michaux } 1988\end{array}$ & NS & $\mathrm{EG}$ & * & * & * & * & e & e \\
\hline Mus musculus L., 1758 & II & & - & - & - & + & + & + \\
\hline \multicolumn{9}{|l|}{ Soricidae } \\
\hline $\begin{array}{l}\text { Crocidura canariensis Hutterer, López-Jurado } \\
\text { and Vogel, } 1987\end{array}$ & NS & ES & - & - & + & - & - & - \\
\hline
\end{tabular}

\begin{tabular}{|c|c|c|c|c|c|c|c|c|}
\hline PHYLUM FUNGI & Or & En & $\mathrm{RE}$ & RO & MC & AL & LG & LO \\
\hline \multicolumn{9}{|l|}{ Agaricaceae } \\
\hline Agaricus aridicola Geml, Geiser and Royse & NP & & - & - & - & - & + & - \\
\hline Montagnea arenaria (DC.) Zeller & NP & & - & - & - & - & + & - \\
\hline Tulostoma brumale Pers. & NP & & - & - & - & - & - & + \\
\hline Tulostoma giovanellae Bres. & NP & & - & - & - & + & - & + \\
\hline \multicolumn{9}{|l|}{ Entolomataceae } \\
\hline Entoloma phaeocyathus Noordel. & NP & & - & - & - & - & - & + \\
\hline \multicolumn{9}{|l|}{ Pezizaceae } \\
\hline Terfezia canariensis Bordallo and Rodríguez & NS & ES & - & - & - & - & + & - \\
\hline \multicolumn{9}{|l|}{ Phelloriniaceae } \\
\hline Phellorinia herculeana (Pers.) Kreisel & NP & & - & - & - & + & - & - \\
\hline \multicolumn{9}{|l|}{ Incertae sedis } \\
\hline Uredo marmoxaiae Speg. & NS & ES & - & - & - & - & + & - \\
\hline PHYLUM BRYOPHYTA & Or & En & $\mathrm{RE}$ & $\mathrm{RO}$ & MC & $\mathrm{AL}$ & LG & LO \\
\hline \multicolumn{9}{|l|}{ Bryaceae } \\
\hline Bryum radiculossum Brid. & NP & & - & - & - & - & - & + \\
\hline $\begin{array}{l}\text { Ptychostomum imbricatulum (Müll. Hal.) D.T. } \\
\text { Holyoak and N. Pedersen }\end{array}$ & NP & & - & - & - & + & - & - \\
\hline \multicolumn{9}{|l|}{ Funariaceae } \\
\hline Entosthodon attenuatus (Dicks.) Bryhn & NP & & - & - & - & - & - & + \\
\hline Entosthodon pulchellus (H. Philib.) Brugués & NP & & - & - & - & + & - & - \\
\hline \multicolumn{9}{|l|}{ Pottiaceae } \\
\hline Aloina ambigua (Bruch and Schimp.) Limpr. & NP & & - & - & - & - & + & - \\
\hline
\end{tabular}




\begin{tabular}{|c|c|c|c|c|c|c|c|}
\hline Didymodon vinealis (Brid.) R.H. Zander & NP & - & - & - & - & - & + \\
\hline Tortella flavovirens (Bruch) Broth. & NP & - & - & + & + & + & + \\
\hline Tortella nitida (Lindb.) Broth. & NP & - & - & - & + & - & - \\
\hline Tortula atrovirens $(\mathrm{Sm}$.$) Lindb.$ & NP & - & - & - & + & + & + \\
\hline Tortula muralis Hedw. & NP & - & - & + & + & + & + \\
\hline Trichostomum brachydontium Bruch & NP & - & - & - & - & - & + \\
\hline
\end{tabular}

\begin{tabular}{|c|c|c|c|c|c|c|c|c|}
\hline VASCULAR PLANTS & $\mathrm{Or}$ & En & $\mathrm{RE}$ & $\mathrm{RO}$ & $\mathrm{MC}$ & $\mathrm{AL}$ & LG & LO \\
\hline \multicolumn{9}{|l|}{ PHYLUM PTERIDOPHYTA } \\
\hline \multicolumn{9}{|l|}{ Adiantaceae } \\
\hline Adiantum capillus-veneris $\mathrm{L}$. & $\mathrm{NO}$ & & - & - & - & + & - & - \\
\hline \multicolumn{9}{|l|}{ Aspleniaceae } \\
\hline Asplenium hemionitis $\mathrm{L}$. & NS & & - & - & - & + & - & - \\
\hline \multicolumn{9}{|l|}{ Ophioglossaceae } \\
\hline Ophioglossum azoricum C. Presl & NS & & - & - & - & - & - & + \\
\hline Ophioglossum polyphyllum A. Braun and Seub. & NS & & - & - & - & - & - & + \\
\hline \multicolumn{9}{|l|}{ PHYLUM SPERMATOPHYTA } \\
\hline \multicolumn{9}{|l|}{ Agavaceae } \\
\hline Agave fourcroydes Lem. & IS & & - & - & - & - & - & + \\
\hline Agave sisalana (Engelm.) Perr. & IS & & - & - & - & - & - & + \\
\hline \multicolumn{9}{|l|}{ Aizoaceae } \\
\hline Aizoon canariense $\mathrm{L}$. & NS & & - & + & + & + & + & + \\
\hline $\begin{array}{l}\text { Carpobrotus edulis (L.) N.E. Br. } \\
\text { in E.P. Phillips }\end{array}$ & II & & - & - & - & - & - & + \\
\hline Mesembryanthemum crystallinum $\mathrm{L}$. & $\mathrm{NO}$ & & + & - & + & + & + & + \\
\hline Mesembryanthemum nodiflorum L. & NO & & + & + & + & + & + & + \\
\hline \multicolumn{9}{|l|}{ Alliaceae } \\
\hline Allium subhirsutum L. & NP & & - & - & + & + & + & - \\
\hline \multicolumn{9}{|l|}{ Amaryllidaceae } \\
\hline Pancratium canariense Ker-Gawl. & NS & ES & - & - & + & - & - & - \\
\hline Pancratium maritimum $\mathrm{L}$. & NP & & - & - & + & - & - & - \\
\hline \multicolumn{9}{|l|}{ Apiaceae } \\
\hline Astydamia latifolia (L. f.) Baill. & NS & & - & - & + & + & + & + \\
\hline Bupleurum semicompositum $\mathrm{L}$. & NS & & - & - & + & + & + & + \\
\hline Petroselinum crispum (Mill.) A.W. Hill & IS & & - & - & - & + & - & - \\
\hline Torilis nodosa (L.) Gaertn. & NO & & - & - & - & - & + & - \\
\hline \multicolumn{9}{|l|}{ Araceae } \\
\hline Arum italicum Mill. & NO & & - & - & - & - & + & - \\
\hline
\end{tabular}




\begin{tabular}{|c|c|c|c|c|c|c|c|c|}
\hline \multicolumn{9}{|l|}{ Asclepiadaceae } \\
\hline Caralluma burchardii N.E. Br. & NS & & - & - & + & - & + & + \\
\hline \multicolumn{9}{|l|}{ Asphodelaceae } \\
\hline Aloe vera (L.) Burm. $\mathrm{f}$. & IS & & - & - & - & - & - & + \\
\hline Asphodelus fistulosus L. & NP & & - & - & + & + & + & + \\
\hline Asphodelus tenuifolius Cav. & NP & & - & - & + & + & + & + \\
\hline \multicolumn{9}{|l|}{ Asteraceae } \\
\hline Andryala pinnatifida Aiton & NS & ES & - & - & - & - & + & + \\
\hline $\begin{array}{l}\text { Argyranthemum maderense (D. Don) } \\
\text { Humphries }\end{array}$ & NS & ES & - & - & - & - & + & - \\
\hline Artemisia thuscula Cav. & NS & ES & - & - & - & - & - & + \\
\hline Asteriscus intermedius (DC.) Pit. and Proust & NS & ES & - & - & + & - & - & - \\
\hline Asteriscus sericeus (L. f.) DC. & NS & ES & - & - & - & - & - & + \\
\hline Atractylis cancellata $\mathrm{L}$. & NP & & - & - & - & + & + & - \\
\hline Calendula aegyptiaca Desf. & NP & & - & - & + & + & + & + \\
\hline Calendula arvensis $\mathrm{L}$. & NO & & - & - & + & + & + & + \\
\hline Centaurea melitensis $\mathrm{L}$. & NO & & - & - & - & - & + & - \\
\hline Chrysanthemum coronarium L. & NO & & - & - & - & + & + & - \\
\hline Filago desertorum Pomel & NP & & - & - & - & + & - & - \\
\hline Filago germanica (L.) Huds. & NO & & - & - & - & - & - & + \\
\hline Filago pyramidata L. & NP & & - & - & - & + & + & + \\
\hline Glebionis coronaria (L.) Spach & IP & & - & - & - & + & + & - \\
\hline Hedypnois rhagadioloides (L.) F.W. Schmidt & NO & & - & - & - & - & - & + \\
\hline Hypochoeris achyrophorus $\mathrm{L}$. & NO & & - & - & + & - & - & - \\
\hline $\begin{array}{l}\text { Ifloga spicata (Forssk.) Sch. Bip. ssp. obovata } \\
\text { (Boll.) Kunk. }\end{array}$ & NO & & - & - & + & + & + & + \\
\hline Kleinia neriifolia Haw. & NS & ES & - & - & + & - & + & + \\
\hline Laphangium luteoalbum (L.) Tzvelev & $\mathrm{NO}$ & & - & - & - & - & - & + \\
\hline Launaea arborescens (Batt.) Murb. & NS & & - & - & + & + & + & + \\
\hline Launaea nudicaulis (L.) Hook. f. & NP & & - & - & + & + & + & + \\
\hline Leontodon taraxacoides (Vill.) Mérat & IP & & - & - & + & + & + & + \\
\hline Otanthus maritimus (L.) Hoffmanns. and Link & NP & & - & - & - & - & + & - \\
\hline Phagnalon purpurascens Sch. Bip. & NP & & - & - & - & - & + & - \\
\hline Phagnalon rupestre (L.) DC. & NP & & - & - & + & + & + & - \\
\hline $\begin{array}{l}\text { Reichardia ligulata (Vent.) G. Kunkel and } \\
\text { Sunding }\end{array}$ & NS & ES & - & - & + & + & - & - \\
\hline Reichardia tingitana (L.) Roth & NP & & - & - & - & - & + & + \\
\hline $\begin{array}{l}\text { Senecio glaucus L. ssp. coronopifolius (Marie) } \\
\text { Alex. }\end{array}$ & NP & & - & - & + & + & + & + \\
\hline
\end{tabular}




\begin{tabular}{|c|c|c|c|c|c|c|c|c|}
\hline Senecio leucanthemifolius Poir. & NP & & - & - & + & + & - & - \\
\hline $\begin{array}{l}\text { Sonchus bourgeaui Sch. Bip. in Webb and } \\
\text { Berthel. }\end{array}$ & NS & & + & - & - & - & + & - \\
\hline Sonchus oleraceus L. & NO & & - & - & + & - & + & + \\
\hline Sonchus pinnatifidus Cav. & NS & & - & - & + & - & + & - \\
\hline $\begin{array}{l}\text { Urospermum picroides (L.) Scop. ex F.W. } \\
\text { Schmidt }\end{array}$ & $\mathrm{NO}$ & & - & - & + & + & + & + \\
\hline \multicolumn{9}{|l|}{ Balanophoraceae } \\
\hline Cynomorium coccineum $\mathrm{L}$. & NP & & - & - & - & - & + & - \\
\hline \multicolumn{9}{|l|}{ Boraginaceae } \\
\hline Arnebia decumbens (Vent.) Coss. and Kralik & NP & & - & - & - & - & + & - \\
\hline Buglossoides arvensis (L.) I.M. Johnst. & NP & & - & - & + & + & + & + \\
\hline Echium bonnetii Coincy & NS & ES & - & - & - & - & - & + \\
\hline Echium lancerottense Lems and Holzapfel & NS & ES & - & - & + & + & + & - \\
\hline Heliotropium ramosissimum (Lehm.) DC. & NS & & - & - & + & + & + & + \\
\hline \multicolumn{9}{|l|}{ Brassicaceae } \\
\hline Cakile maritima Scop. & NP & & - & - & - & - & + & + \\
\hline Capsella bursa-pastoris (L.) Medik. & $\mathrm{NO}$ & & - & - & - & - & - & + \\
\hline Carrichtera annua (L.) DC. & NP & & - & - & + & - & + & + \\
\hline Erucastrum canariense Webb and Berthel. & NS & ES & - & - & - & - & + & - \\
\hline $\begin{array}{l}\text { Lobularia canariensis (DC.) L. Borgen ssp. } \\
\text { marginata (Webb) L. Borgen }\end{array}$ & NS & & - & - & - & - & + & - \\
\hline Lobularia libyca (Viv.) C.F.W. Meissn. & NP & & - & - & - & - & - & + \\
\hline Matthiola bolleana Webb ex Christ & $\mathrm{NO}$ & ES & - & - & - & - & + & - \\
\hline $\begin{array}{l}\text { Matthiola parviflora (Schousb.) R. Br. } \\
\text { in W.T. Aiton }\end{array}$ & NP & & - & - & - & - & + & - \\
\hline Notoceras bicorne (Aiton) Amo & NP & & - & - & + & + & + & + \\
\hline Sinapis arvensis $\mathrm{L}$. & $\mathrm{NO}$ & & - & - & + & - & + & - \\
\hline Sisymbrium erysimoides Desf. & $\mathrm{NO}$ & & - & - & - & + & + & + \\
\hline \multicolumn{9}{|l|}{ Cactaceae } \\
\hline Minuartia geniculata (Poir.) Thell. & NP & & - & - & - & + & + & + \\
\hline Opuntia dillenii (Ker-Gawl.) Haw. & II & & - & - & - & - & - & + \\
\hline Opuntia maxima Mill. & II & & - & - & - & - & + & + \\
\hline Opuntia tomentosa Salm-Dyck & II & & - & - & - & - & - & + \\
\hline \multicolumn{9}{|l|}{ Campanulaceae } \\
\hline Campanula erinus L. & $\mathrm{NO}$ & & - & - & - & - & - & + \\
\hline Wahlenbergia lobelioides (L. f.) Link & NS & & - & - & - & + & + & - \\
\hline \multicolumn{9}{|l|}{ Caryophyllaceae } \\
\hline Arenaria leptoclados (Rchb.) Guss. & NP & & - & - & - & - & + & - \\
\hline
\end{tabular}




\begin{tabular}{|c|c|c|c|c|c|c|c|c|}
\hline Herniaria cinerea DC. in Lam. and DC. & NP & & - & - & + & + & + & + \\
\hline Minuartia geniculata (Poir.) Thell. & NP & & - & - & + & + & + & - \\
\hline Polycarpaea divaricata (Aiton) Poir. & NS & ES & - & - & - & - & + & - \\
\hline Polycarpaea nivea (Aiton) Webb & NS & & - & - & + & + & + & + \\
\hline Polycarpon tetraphyllum (L.) L. & $\mathrm{NO}$ & & - & - & + & + & + & - \\
\hline Pteranthus dichotomus Forssk. & NP & & - & - & - & - & - & + \\
\hline Silene apetala Willd. & $\mathrm{NO}$ & & - & - & - & + & + & + \\
\hline Silene nocturna L. & $\mathrm{NO}$ & & - & - & - & - & + & - \\
\hline $\begin{array}{l}\text { Spergularia bocconei (Scheele) Graebn. in Asch. } \\
\text { and Graebn. }\end{array}$ & NO & & - & - & - & - & + & + \\
\hline Spergularia diandra (Guss.) Heldreich & NO & & - & - & - & - & + & + \\
\hline $\begin{array}{l}\text { Spergularia fallax (Lowe) E.H.L. Krause } \\
\text { in Sturm }\end{array}$ & NP & & - & - & + & + & - & + \\
\hline Spergularia fimbriata Boiss. and Reut. & NP & & - & - & + & + & - & - \\
\hline Spergularia media (L.) C. Presl & IP & & - & - & + & + & + & + \\
\hline Stellaria media (L.) Vill. & IP & & - & - & - & - & - & + \\
\hline \multicolumn{9}{|l|}{ Chenopodiaceae } \\
\hline $\begin{array}{l}\text { Arthrocnemum macrostachyum (Moric.) } \\
\text { K. Koch }\end{array}$ & NS & & - & - & - & + & + & + \\
\hline $\begin{array}{l}\text { Atriplex glauca L. ssp. ifniensis (Caball.) } \\
\text { Rivas-Mart. and al. }\end{array}$ & NP & & - & - & + & + & + & + \\
\hline Atriplex halimus L. & NP & & - & - & - & - & + & + \\
\hline Beta macrocarpa Guss. & NP & & - & - & - & + & - & - \\
\hline Chenoleoides tomentosa (Lowe) Botsch. & NS & & + & - & + & + & + & + \\
\hline Chenopodium album L. & NO & & - & - & - & - & + & - \\
\hline Chenopodium ambrosioides L. & IS & & - & - & - & + & + & - \\
\hline Chenopodium murale L. & IP & & + & - & + & + & + & + \\
\hline $\begin{array}{l}\text { Patellifolia patellaris (Moq.) A.J. Scott, } \\
\text { Ford-Lloyd and J. T. Williams }\end{array}$ & NS & & + & - & + & + & + & + \\
\hline $\begin{array}{l}\text { Patellifolia procumbens (C. Sm. ex Hornem.) } \\
\text { A.J. Scott, Ford-Lloyd and J.T. Williams }\end{array}$ & NS & & - & - & + & + & + & + \\
\hline $\begin{array}{l}\text { Patellifolia webbiana (Moq.) A.J. Scott, } \\
\text { Ford-Lloyd and J.T. Williams }\end{array}$ & NS & ES & - & - & - & + & + & + \\
\hline Salsola divaricata Masson ex Link in Buch & NS & ES & + & + & + & + & + & + \\
\hline Salsola tetrandra Forssk. & NS & & - & - & + & + & + & + \\
\hline Salsola vermiculata $\mathrm{L}$. & NS & & - & - & + & + & + & + \\
\hline Sarcocornia perennis (Mill.) A.J. Scott & NS & & - & - & - & - & - & + \\
\hline Suaeda fruticosa Forssk. ex J.F. Gmelin & IS & & - & - & - & - & - & + \\
\hline Suaeda ifniensis Caball. in Maire & NP & & + & - & + & - & - & + \\
\hline Suaeda maritima (L.) Dumort. & NS & & - & - & + & + & - & - \\
\hline
\end{tabular}




\begin{tabular}{|c|c|c|c|c|c|c|c|c|}
\hline Suaeda mollis Delile & NS & & - & - & - & - & - & - \\
\hline Suaeda vera Forssk. ex J.F. Gmel. & NS & & - & + & - & + & + & + \\
\hline Traganum moquinii Webb ex Moq. in DC. & NS & & - & - & - & - & + & + \\
\hline \multicolumn{9}{|l|}{ Cistaceae } \\
\hline Helianthemum canariense (Jacq.) Pers. & NS & & - & - & + & - & + & + \\
\hline \multicolumn{9}{|l|}{ Convallariaceae } \\
\hline Asparagus arborescens Willd. & NS & ES & - & - & - & - & + & - \\
\hline Asparagus nesiotes Svent. & NS & & - & - & + & - & + & - \\
\hline \multicolumn{9}{|l|}{ Convolvulaceae } \\
\hline Convolvulus althaeoides L. & $\mathrm{NO}$ & & - & - & - & - & - & + \\
\hline Convolvulus siculus L. & $\mathrm{NO}$ & & - & - & - & - & + & - \\
\hline \multicolumn{9}{|l|}{ Crassulaceae } \\
\hline Aeonium arboreum (L.) Webb and Berthel. & NS & ES & - & - & - & - & - & + \\
\hline Aeonium lancerottense (Praeger) Praeger & NS & ES & - & - & - & - & - & + \\
\hline Aeonium nobile (Praeger) Praeger & NS & ES & - & - & - & - & - & + \\
\hline Umbilicus gaditanus Boiss. & NP & & - & - & + & + & + & + \\
\hline \multicolumn{9}{|l|}{ Cuscutaceae } \\
\hline Cuscuta planiflora Ten. & NP & & - & - & - & + & + & + \\
\hline \multicolumn{9}{|l|}{ Cyperaceae } \\
\hline Cyperus capitatus Vand. & NS & & - & - & - & - & + & + \\
\hline Cyperus laevigatus L. & NP & & - & - & - & + & - & - \\
\hline Cyperus rotundus $\mathrm{L}$. & IS & & - & - & - & - & - & + \\
\hline \multicolumn{9}{|l|}{ Euphorbiaceae } \\
\hline Euphorbia balsamifera Aiton & NS & & - & - & + & + & + & + \\
\hline Euphorbia paralias L. & NS & & - & - & - & - & + & + \\
\hline Euphorbia peplus L. & $\mathrm{NO}$ & & - & - & - & - & + & + \\
\hline Euphorbia regis-jubae Webb. and Berthel. & NS & & - & - & + & + & + & + \\
\hline Euphorbia terracina $\mathrm{L}$. & NP & & - & - & - & + & - & - \\
\hline Mercurialis annua L. & IP & & - & - & + & + & + & + \\
\hline Ricinus communis L. & II & & - & - & - & - & - & + \\
\hline \multicolumn{9}{|l|}{ Fabaceae } \\
\hline Astragalus hamosus L. & NS & & - & - & + & + & + & + \\
\hline Bituminaria bituminosa (L.) C.H. Stirt. & NP & & - & - & + & + & - & - \\
\hline Coronilla viminalis Salisb. & NP & & - & - & + & - & - & - \\
\hline Lathyrus sativus L. & IS & & - & - & - & - & + & - \\
\hline Lotus glinoides Delile & NP & & - & - & + & + & + & + \\
\hline Lotus lancerottensis Webb and Berthel. & NS & & - & - & + & + & + & + \\
\hline
\end{tabular}




\begin{tabular}{|c|c|c|c|c|c|c|c|c|}
\hline Medicago laciniata (L.) Mill. & NS & & - & - & + & + & + & + \\
\hline Medicago littoralis Rohde ex Loisel. & NS & & - & - & + & + & - & + \\
\hline Medicago polymorpha L. & NO & & - & - & - & + & + & - \\
\hline Ononis hebecarpa Webb and Berthel. & NS & ES & - & - & - & - & + & + \\
\hline $\begin{array}{l}\text { Ononis hesperia (Maire) H. Förther } \\
\text { and D. Podlech }\end{array}$ & NS & & - & - & - & - & + & - \\
\hline Ononis laxiflora Desf. & NP & & - & - & - & + & - & - \\
\hline Ononis pendula Desf. & NS & & - & - & - & + & - & - \\
\hline Ononis serrata Forssk. & $\mathrm{NO}$ & & - & - & + & - & + & + \\
\hline Trigonella stellata Forssk. & NS & & - & - & + & + & + & + \\
\hline \multicolumn{9}{|l|}{ Frankeniaceae } \\
\hline Frankenia boissieri Reut. ex Boiss. & NS & & - & - & - & - & - & + \\
\hline Frankenia capitata Webb and Berthel. & NS & & - & - & - & + & - & + \\
\hline Frankenia ericifolia C. Sm. ex DC. & NS & & - & + & + & + & + & + \\
\hline Frankenia pulverulenta L. & NP & & - & - & + & + & + & - \\
\hline \multicolumn{9}{|l|}{ Fumariaceae } \\
\hline Fumaria bastardii Boreau & $\mathrm{NO}$ & & - & - & - & - & + & + \\
\hline Fumaria muralis Sonder ex Koch & NP & & - & - & - & + & - & - \\
\hline \multicolumn{9}{|l|}{ Geraniaceae } \\
\hline Erodium botrys (Cav.) Bertol. & $\mathrm{NO}$ & & - & - & - & - & + & + \\
\hline Erodium chium (L.) Willd. & $\mathrm{NO}$ & & - & - & + & + & + & + \\
\hline Erodium cicutarium (L.) L'Hér. in Aiton & NO & & - & - & - & - & + & + \\
\hline Erodium laciniatum (Cav.) Willd. & $\mathrm{NO}$ & & - & - & - & - & + & - \\
\hline Erodium malacoides (L.) L'Hér. in Aiton & $\mathrm{NO}$ & & - & - & - & + & + & + \\
\hline Erodium neuradifolium Delile & NP & & - & - & + & - & + & - \\
\hline \multicolumn{9}{|l|}{ Hyacinthaceae } \\
\hline Scilla dasyantha Webb and Berthel. & NS & ES & - & - & - & - & - & + \\
\hline \multicolumn{9}{|l|}{ Lamiaceae } \\
\hline Ajuga iva (L.) Schreb. & NP & & - & - & + & + & + & + \\
\hline $\begin{array}{l}\text { Micromeria varia Benth. ssp. rupestris } \\
\text { (Webb and Berthel.) P. Pérez }\end{array}$ & NS & & - & - & + & + & - & - \\
\hline Salvia aegyptiaca L. & NS & & - & - & - & - & - & + \\
\hline \multicolumn{9}{|l|}{ Liliaceae } \\
\hline Asparagus nesiotes Svent. & NS & & - & - & + & - & + & - \\
\hline $\begin{array}{l}\text { Allium subhirsutum L. ssp. obtusitepalum } \\
\text { (Svent.) G. Kunkel }\end{array}$ & NS & ESS & - & - & + & + & + & - \\
\hline Androcymbium psammophilum Svent. & NS & ES & - & - & - & - & - & + \\
\hline Dipcadi serotinum (L.) Medik. & NP & & - & - & + & + & + & + \\
\hline
\end{tabular}




\begin{tabular}{|c|c|c|c|c|c|c|c|c|}
\hline Linaceae & & & & & & & & \\
\hline Linum strictum $\mathrm{L}$. & NP & & - & - & - & + & - & - \\
\hline Malvaceae & & & & & & & & \\
\hline Malva parviflora $\mathrm{L}$. & $\mathrm{NO}$ & & - & - & - & + & + & + \\
\hline Onagraceae & & & & & & & & \\
\hline Oenothera rosea L'Hér. ex Aiton & IS & & - & - & - & - & - & + \\
\hline Orobanchaceae & & & & & & & & \\
\hline Cistanche phelipaea (L.) Cout. & NP & & - & - & + & + & + & + \\
\hline Orobanche minor Sm. & NP & & - & - & - & - & + & - \\
\hline $\begin{array}{l}\text { Phelipanche gratiosa (Webb) Carlón, } \\
\text { G. Gómez, M. Laínz, Moreno Mor., } \\
\text { Ó. Sánchez and Schneew. }\end{array}$ & NS & ES & - & - & - & - & + & - \\
\hline Oxalidaceae & & & & & & & & \\
\hline Oxalis corniculata $\mathrm{L}$. & $\mathrm{NO}$ & & - & - & - & - & - & + \\
\hline Oxalis pes-caprae Ker-Gawl. & II & & - & - & - & - & - & + \\
\hline Papaveraceae & & & & & & & & \\
\hline Papaver dubium L. & $\mathrm{NO}$ & & - & - & - & + & + & - \\
\hline Plantaginaceae & & & & & & & & \\
\hline Plantago afra $\mathrm{L}$. & $\mathrm{NO}$ & & - & - & - & + & + & + \\
\hline Plantago coronopus L. & $\mathrm{NP}$ & & - & - & + & + & + & + \\
\hline Plantago ovata Forssk. & NP & & - & - & + & + & + & + \\
\hline Plantago phaeostoma Boiss. and Heldr. & $\mathrm{NO}$ & & - & - & + & + & - & - \\
\hline Plumbaginaceae & & & & & & & & \\
\hline Limonium ovalifolium ssp. canariensis Pignatti & NS & IE & - & - & - & - & - & + \\
\hline $\begin{array}{l}\text { Limonium papillatum (Webb and Berthel.) } \\
\text { Kuntze }\end{array}$ & NS & & - & - & + & + & + & + \\
\hline Limonium puberulum (Webb) Kuntze & NS & ES & - & - & - & - & + & - \\
\hline Limonium tuberculatum (Boiss.) Kuntze & NS & & - & - & - & - & - & + \\
\hline Poaceae & & & & & & & & \\
\hline Agrostis castellana Boiss. and Reut. & $\mathrm{NO}$ & & - & - & - & - & - & + \\
\hline Anisantha rigida (Roth) Hyl. & $\mathrm{NO}$ & & - & - & - & + & + & - \\
\hline Anisantha rubens (L.) & NS & & - & - & + & + & + & - \\
\hline Arundo donax $\mathrm{L}$ & II & & - & - & - & - & - & + \\
\hline Avena fatua L. ssp. meridionalis Malzev & NS & & - & - & - & - & + & - \\
\hline Avena sterilis $\mathrm{L}$. & $\mathrm{NO}$ & & - & - & - & - & + & - \\
\hline Bromus rigidus Roth & $\mathrm{NO}$ & & - & - & - & + & + & - \\
\hline Castellia tuberculosa (Moris) Bor & $\mathrm{NO}$ & & - & - & + & + & + & - \\
\hline Cenchrus ciliaris $\mathrm{L}$. & NP & & - & - & - & - & + & + \\
\hline Ceratochloa cathartica (Vall) Herter & IS & & - & - & - & - & - & + \\
\hline
\end{tabular}




\begin{tabular}{|c|c|c|c|c|c|c|c|c|}
\hline Enneapogon desvauxii P. Beauv. & NO & & - & - & - & + & + & + \\
\hline Eragrostis barrelieri Daveau & $\mathrm{NO}$ & & - & - & + & + & + & + \\
\hline Hordeum marinum Huds. & NO & & - & - & - & - & - & + \\
\hline Hordeum murinum L. & $\mathrm{NO}$ & & - & - & - & - & + & + \\
\hline Hordeum vulgare $\mathrm{L}$. & IS & & - & - & - & - & - & + \\
\hline Lamarckia aurea (L.) Moench & NP & & - & - & + & + & + & + \\
\hline Lolium perenne L. & IP & & - & - & - & - & - & + \\
\hline Lolium rigidum Gaudin & $\mathrm{NO}$ & & - & - & - & - & - & + \\
\hline Ochlopoa annua (L.) H. Scholz & $\mathrm{NO}$ & & - & - & - & - & - & + \\
\hline Phalaris canariensis L. & NP & & - & - & + & + & + & + \\
\hline Phalaris minor Retz. & NP & & - & - & - & + & + & - \\
\hline Piptatherum miliaceum (L.) Coss. & $\mathrm{NO}$ & & - & - & - & - & - & - \\
\hline Rostraria cristata (L.) Tzvelev & NP & & - & - & - & + & + & - \\
\hline Rostraria pumila (Desf.) Tzvelev & NP & & - & - & - & + & + & + \\
\hline Schismus arabicus Nees & NO & & - & - & - & - & - & + \\
\hline Schismus barbatus (L.) Thell. & NO & & - & - & + & + & + & + \\
\hline Sphenopus divaricatus (Gouan) Rchb. & $\mathrm{NO}$ & & - & - & - & - & - & + \\
\hline Stipa capensis Thunb. & NP & & - & - & - & + & + & + \\
\hline Tetrapogon villosus Desf. & NP & & - & - & - & + & + & + \\
\hline Trachynia distachya (L.) Link & $\mathrm{NO}$ & & - & - & - & + & + & + \\
\hline Tragus racemosus (L.) All. & NP & & - & - & - & + & - & - \\
\hline Trisetaria panicea (Lam.) Paunero & $\mathrm{NO}$ & & - & - & - & + & - & - \\
\hline \multicolumn{9}{|l|}{ Polygonaceae } \\
\hline Emex spinosa (L.) Campd. & NP & & - & - & + & + & + & + \\
\hline Polygonum maritimum L. & NS & & - & - & - & - & + & - \\
\hline Rumex lunaria L. & NS & ES & - & - & - & - & - & + \\
\hline Rumex vesicarius $\mathrm{L}$. & NP & & - & - & + & + & + & + \\
\hline \multicolumn{9}{|l|}{ Portulacaceae } \\
\hline Portulaca oleracea L. & IS & & - & - & - & - & + & - \\
\hline \multicolumn{9}{|l|}{ Primulaceae } \\
\hline Anagallis arvensis $\mathrm{L}$. & NO & & - & - & + & + & + & + \\
\hline Asterolinon linum-stellatum (L.) Duby in DC. & NP & & - & - & - & - & + & + \\
\hline \multicolumn{9}{|l|}{ Ranunculaceae } \\
\hline Adonis microcarpa DC. & IP & & - & - & - & - & + & - \\
\hline \multicolumn{9}{|l|}{ Resedaceae } \\
\hline Oligomeris linifolia (Vahl) J.F. Macbr. & NS & & - & - & + & + & + & + \\
\hline Reseda crystallina Webb and Berthel. & NS & ES & - & - & + & + & + & + \\
\hline
\end{tabular}




\begin{tabular}{|c|c|c|c|c|c|c|c|c|}
\hline \multicolumn{9}{|l|}{ Rubiaceae } \\
\hline Galium aparine L. & $\mathrm{NO}$ & & - & - & - & + & - & - \\
\hline Rubia fruticosa Aiton & NS & & + & - & + & - & - & - \\
\hline \multicolumn{9}{|l|}{ Santalaceae } \\
\hline Thesium humile Vahl & NP & & - & - & - & - & - & + \\
\hline \multicolumn{9}{|l|}{ Scrophulariaceae } \\
\hline Kickxia sagittata (Poir.) Rothm. & NS & & - & - & + & - & + & + \\
\hline Misopates orontium (L.) Raf. & NP & & - & - & + & + & + & + \\
\hline Scrophularia arguta Sol. ex Aiton & NS & & - & - & + & + & + & + \\
\hline \multicolumn{9}{|l|}{ Solanaceae } \\
\hline Lycium intricatum Boiss. & NS & & + & - & + & + & + & + \\
\hline Lycopersicon esculentum Mill. & IS & & - & - & - & - & + & + \\
\hline Nicotiana glauca R.C. Graham & II & & - & - & + & + & + & + \\
\hline Solanum nigrum $\mathrm{L}$. & $\mathrm{NO}$ & & - & - & - & + & + & + \\
\hline \multicolumn{9}{|l|}{ Urticaceae } \\
\hline Forsskaolea angustifolia Retz. & NS & ES & - & - & + & + & + & + \\
\hline Parietaria debilis G. Forst. & NP & & - & - & - & - & - & + \\
\hline \multicolumn{9}{|l|}{ Verbenaceae } \\
\hline Verbena supina $\mathrm{L}$. & IP & & - & - & - & - & - & + \\
\hline \multicolumn{9}{|l|}{ Zygophyllaceae } \\
\hline Fagonia cretica L. & NP & & - & - & + & + & + & + \\
\hline $\begin{array}{l}\text { Tetraena fontanesii (Webb and Berthel.) Beier } \\
\text { and Thulin }\end{array}$ & NP & & + & + & + & + & + & + \\
\hline Phylum Arthropoda & Or & En & $\mathrm{RE}$ & $\mathrm{RO}$ & MC & AL & LG & $\mathrm{LO}$ \\
\hline \multicolumn{9}{|l|}{ ORDER POLYXENIDA } \\
\hline \multicolumn{9}{|l|}{ Polyxenidae } \\
\hline $\begin{array}{l}\text { Macroxenus enghoffi Nguyen Duy-Jacquemin, } \\
1996\end{array}$ & NP & & - & * & + & - & - & - \\
\hline \multicolumn{9}{|l|}{ ORDER JULIDA } \\
\hline \multicolumn{9}{|l|}{ Julidae } \\
\hline Dolichoiulus wunderlichi Enghoff, 1992 & NS & ES & - & * & + & + & - & - \\
\hline \multicolumn{9}{|l|}{ ORDER ISOPODA } \\
\hline \multicolumn{9}{|l|}{ Armadillidae } \\
\hline Eluma caelata Miers, 1885 & II & & - & - & + & - & - & - \\
\hline Venezillo canariensis (Dollfus, 1893) & NS & ES & - & * & + & - & + & - \\
\hline \multicolumn{9}{|l|}{ Halophilosciidae } \\
\hline Halophiloscia couchi Kinahan, 1858 & IP & & - & * & + & - & + & - \\
\hline
\end{tabular}




\section{Ligiidae}

Ligia italica Fabricius, 1798

IP

\section{Platyarthridae}

Trichorhina anophthalma Arcangeli, 1935

Porcellionidae

Leptotrichus panzeri Audouin, 1826 IS

Porcellio alluaudi Dolfus, $1893 \quad$ NO

Porcellio lamellatus Budde-Lund, 1885

Porcellio spinipes Dollfus, 1893

Soteriscus disimilis Rodríguez, 1990

NS ES

\section{Tylidae}

Tylos latreillei Audouin, 1825

IP

Tylos ponticus Grebnitzky, 1874 IP

ORDER SOLIFUGA

\section{Karschiidae}

Eusimonia wunderlichi Pieper, 1977

NS ES

ORDER ARANEAE

\section{Araneidae}

Cyclosa insulana Costa, 1834

Nemoscolus laurae (Simon, 1868)

Neoscona crucifera Lucas, 1839

Neoscona subfusca (Koch, 1837)

$\begin{array}{lllllll}\mathrm{NP} & - & * & - & + & - & - \\ \mathrm{NP} & * & * & * & * & * & + \\ \mathrm{NO} & - & * & - & - & + & - \\ \mathrm{NO} & * & * & * & * & * & +\end{array}$

\section{Dictynidae}

Dictyna fuerteventurensis Schmidt, 1976

Nigma puella (Simon, 1870)

$\begin{array}{llllllll}\text { NS } & \text { ES } & - & * & - & + & - & - \\ \text { NS } & & * & * & * & * & * & +\end{array}$

\section{Dysderidae}

Dysdera alegranzaensis Wunderlich, 1992

Dysdera lancerotensis Simon, 1907

Dysdera nesiotes Simon, 1907

$\begin{array}{ll}\text { NS } & \text { IE } \\ \text { NS } & \text { ES } \\ \text { NS } & \text { ES }\end{array}$

\section{Filistatidae}

Filistata canariensis Schmidt, 1976

$$
\text { NS }
$$

\section{Gnaphosidae}

Drassodes lutescens Koch, 1839

Drassodes riedeli Schmidt, 1968

Haplodrassus minor Cambridge, 1879

NO

Oecobius navus Blackwall, 1859

IS

Scotognapha convexa (Simon, 1883)

NS ES




\begin{tabular}{|c|c|c|c|c|c|c|c|c|}
\hline $\begin{array}{l}\text { Scotognapha haria Platnick, Ovtsharenko } \\
\text { and Murphy, } 2001\end{array}$ & NS & ES & - & - & + & - & - & - \\
\hline Scotophaeus musculus Simon, 1878 & NO & & - & * & - & + & - & - \\
\hline Setaphis parvula Lucas, 1846 & NS & & - & - & + & - & - & - \\
\hline Setaphis walteri Platnick and Murphy, 1996 & NS & ES & * & * & * & * & * & + \\
\hline Zelotes scrutatus O. P. Cambridge, 1879 & NP & & - & - & + & - & - & - \\
\hline \multicolumn{9}{|l|}{ Heteropodidae } \\
\hline Cerbalus alegranzaensis Wunderlich, 1992 & NS & IE & - & * & - & + & - & - \\
\hline \multicolumn{9}{|l|}{ Idiopidae } \\
\hline Titanidiops canariensis Wunderlich, 1992 & NS & ES & - & * & - & - & + & - \\
\hline \multicolumn{9}{|l|}{ Linyphiidae } \\
\hline Styloctetor romanus Cambridge, 1872 & NO & & - & * & - & + & - & - \\
\hline \multicolumn{9}{|l|}{ Oecobiidae } \\
\hline Oecobius furcula Wunderlich, 1992 & NS & ES & - & * & - & + & - & - \\
\hline Oecobius navus Blackwall, 1859 & IS & & - & - & - & - & - & + \\
\hline Uroctea paivai (Blackwall, 1868) & NS & ES & - & - & - & - & - & + \\
\hline \multicolumn{9}{|l|}{ Oonopidae } \\
\hline Orchestina pavesii Simon, 1873 & $\mathrm{NO}$ & & - & - & + & - & - & - \\
\hline \multicolumn{9}{|l|}{ Palpimanidae } \\
\hline Palpimanus canariensis Kulczynski, 1909 & NS & ES & - & $*$ & + & + & + & - \\
\hline \multicolumn{9}{|l|}{ Philodromidae } \\
\hline Philodromus pulchellus Lucas, 1846 & $\mathrm{NO}$ & & * & * & * & * & * & + \\
\hline Philodromus punctigerus O.P.-Cambridge, 1908 & NS & ES & - & * & - & + & - & - \\
\hline Thanatus arenicola (Schmidt, 1976) & NS & ES & - & * & + & + & + & + \\
\hline Thanatus fabricii (Audouin, 1826) & NO & & * & $*$ & * & * & * & + \\
\hline Thanatus vulgaris Simon, 1870 & NO & & - & * & - & - & + & - \\
\hline Zimirina hirsuta Cooke, 1964 & NS & ES & - & * & + & + & - & - \\
\hline \multicolumn{9}{|l|}{ Salticidae } \\
\hline Aelurillus lucasi Roewer, 1951 & $\mathrm{NO}$ & & - & * & - & + & - & - \\
\hline Cyrba algerina Lucas, 1846 & NO & & - & - & + & - & - & - \\
\hline $\begin{array}{l}\text { Heliophanus fuerteventurae Schmidt and } \\
\text { Krause, } 1996\end{array}$ & NS & ES & - & - & + & - & - & - \\
\hline $\begin{array}{l}\text { Macaroeris albosignata Schmidt and Krause, } \\
1996\end{array}$ & NS & ES & - & - & + & - & - & - \\
\hline Macaroeris moebii Böesenberg, 1895 & NO & & - & * & - & + & - & - \\
\hline Salticus alegranzaensis Wunderlich, 1995 & NS & ES & - & * & + & + & - & - \\
\hline Yllenus albifrons Lucas, 1846 & NP & & - & - & + & - & - & - \\
\hline Yllenus gavdos Logunov and Marusik, 2003 & NP & & - & * & + & - & - & - \\
\hline
\end{tabular}




\section{Scytodidae}

Scytodes tenerifensis Wunderlich, 1987

NS ES

\section{Segestriidae}

Ariadna canariensis Wunderlich, 1995

Segestria bavarica Koch, 1843

NS ES

\section{Sicariidae}

Loxosceles rufescens Dufour, 1820

IP

\section{Theridiidae}

Eurypoena tuberosa ssp. alegranzaensis

Wunderlich, 1992

Kochiura aulica (C.L. Koch, 1838)

Latrodectus tredecimguttatus (Rossi, 1790)

NS EG

Paidiscura orotavensis Schimidt, 1968

Steatoda grossa Koch, 1838

Steatoda nobilis Thorell, 1875

$\mathrm{NO}$

NO

NS

NO

\section{Thomisidae}

Thomisus onustus Walckenaer, 1805

$\mathrm{NO}$

\section{Uloboridae}

Uloborus parvulus Schmidt, 1976

NS ES

\section{Zodariidae}

Zodarion nesiotes Denis, 1965

Zodarion nesiotioides Wunderlich, 1992

$\begin{array}{llllllll}\text { NS } & \text { ES } & - & - & + & - & - & - \\ \text { NS } & \text { ES } & - & * & + & - & - & -\end{array}$

ORDER LITHOBIOMORPHA

\section{Lithobiidae}

Lithobius crassipes L. Koch, 1862

NO

Lithobius tenerifae Latzel, 1895

NS

ORDER SCOLOPENDROMORPHA

\section{Cryptopidae}

Cryptops trisulcatus Brölemann, 1902

NO

\section{Scolopendridae}

Scolopendra valida Lucas, 1840

NO

ORDER GEOPHILOMORPHA

\section{Geophilidae}

Dignathodon microcephalus Lucas, 1846 NP

Pachymerium ferrugineum C.L. Koch, 1835

NO

\section{Schendilidae}

Nannophilus eximius Meinert, 1870 
ORDER ZYGENTOMA

\section{Lepismatidae}

Ctenolepisma lineata Fabricius, 1775

$\mathrm{NO}$

Ctenolepisma rodriguezi Mendes, Molero,

Bach and Gaju, 1993

Ctenolepisma vieirai Mendes, 1981

NS ES

Neoasterolepisma myrmecobia Silvestri, 1908

NP

ORDER ODONATA

\section{Aeschnidae}

Anax imperator Leach, 1815

NS

Hemianax ephippiger Burmeister, 1839

NS

\section{Coenagriidae}

Ischnura sahariensis Aguesse, 1968

NS

Libellulidae

Sympetrum fonscolombei Sélys, 1840

NS

ORDER MANTODEA

\section{Mantidae}

Pseudoyersinia betancuriae Wiemers

NS ES

ORDER ORTHOPTERA

\section{Acrididae}

Dericorys lobata, 1840

NP

Schistocerca gregaria Forskal, 1775

Sphingonotus pachecoi Bolívar, 1908

Sphingonotus savignyi Saussure, 1884

NP

NP

\section{Gryllidae}

Gryllus bimaculatus De Geer, 1773

IP

Pseudomogoplistes squamiger Fischer, 1843

NP

Pamphagidae

Purpuraria erna Enderlein, 1929

NS EG

ORDER PODUROMORPHA

\section{Hypogastruridae}

Xenylla brevisimilis Stach, 1949

$\mathrm{NO}$

ORDER ENTOMOBRYOMORPHA

\section{Entomobryidae}

Pseudosinella canariensis Gama, 1974

NS

Seira dinizi Gama, 1988

$\mathrm{NO}$ 
ORDER HEMIPTERA

\section{Anthocoridae}

Orius laevigatus Fieber, 1860

NP

Orius niger Wolff, 1811

NP

\section{Corixidae}

Corixa affinis Leach, 1817

NP

Sigara lateralis Leach, 1817

NP

Cydnidae

Byrsinus laticollis (Wagner, 1954)

$\mathrm{NO}$

Cydnus aterrimus Förster, 1771

$\mathrm{NO}$

Macroscytus brunneus Fabricius, 1803

$\mathrm{NO}$

\section{Flatidae}

Cyphopterum posterius Lindberg, 1965

Cyphopterum salinum Lindberg, 1954

NS ES

Lygaeidae

Aphanus rolandri Linnaeus, 1758

Camptocera glaberrima Walker, 1872

NS ES

Geocoris megacephalus Rossi, 1790

Geocoris phaeopterus Germar, 1837

Gonianotus galactodermus Fieber, 1861

$\mathrm{NO}$

Nysius cymoides Spinola, 1840

$\mathrm{NO}$

Nysius thymi ssp. latus Wagner 1958

Spilostethus pandurus Scopoli, 1763

Stygnocoris subglaber (Puton, 1889)

Tropistethus seminitens Puton, 1889

NP

NS

NO

Miridae

Compsonannus longiceps (Wagner, 1965)

Deraeocoris martini (Puton, 1887)

Megacoelum zollikoferiae Lindberg, 1954

Pastocoris putonii (Reuter, 1875)

Phytocoris meinanderi Wagner, 1968

NO

Myrmeleontidae

Acanthaclisis baetica Rambur, 1842

NP

Notonectidae

Anisops sardeus Herrich-Schäffer, 1850

NP

Pentatomidae

Brachynema cinctum (Fabricius, 1775)

$\mathrm{NO}$

$\begin{array}{llllllll}\text { NS } & \text { ES } & - & * & - & - & + & - \\ \text { NO } & & * & * & * & * & * & + \\ \text { NO } & & - & - & + & - & - & - \\ \text { NO } & & - & - & + & - & - & + \\ \text { NS } & \text { ES } & - & * & - & + & + & -\end{array}$

NO

NO

NO

NS ES

NO

ES




\begin{tabular}{|c|c|c|c|c|c|c|c|c|}
\hline Piesmatidae & & & & & & & & \\
\hline Parapiesma rotundatum (Horváth, 1906) & $\mathrm{NO}$ & & - & - & + & - & - & - \\
\hline Psyllidae & & & & & & & & \\
\hline Diaphorina continua Loginova, 1972 & $\mathrm{NO}$ & & $*$ & * & $*$ & $*$ & $*$ & + \\
\hline Saldidae & & & & & & & & \\
\hline Saldula palustris Douglas, 1874 & $\mathrm{NO}$ & & - & * & - & + & - & - \\
\hline Tingidae & & & & & & & & \\
\hline Dictyonota atlantica Péricart, 1981 & NS & ES & - & * & + & + & - & - \\
\hline Tingis ajugarum Frey-Gessner, 1872 & NP & & - & * & - & + & - & - \\
\hline ORDER PLANIPENNIA & & & & & & & & \\
\hline Chrysopidae & & & & & & & & \\
\hline Chrysoperla carnea (Stephens, 1836) & $\mathrm{NO}$ & & - & - & + & - & - & - \\
\hline Coniopterygidae & & & & & & & & \\
\hline Semidalis candida Navás, 1916 & NP & & * & * & * & * & * & + \\
\hline Hemerobiidae & & & & & & & & \\
\hline Wesmaelius navasi (Andreu, 1911) & NP & & $*$ & * & $*$ & $*$ & $*$ & + \\
\hline ORDER COLEOPTERA & & & & & & & & \\
\hline Anobiidae & & & & & & & & \\
\hline Clada oromii Español, 1978 & NS & & * & * & + & * & * & - \\
\hline Dignomus gracilipes Wollaston, 1862 & NS & ES & * & * & * & * & * & + \\
\hline Hyperisus filicorne (Israelon, 1974) & NS & ES & * & * & + & * & * & - \\
\hline Lasioderma latitans (Wollaston, 1861) & IP & & * & * & + & * & * & - \\
\hline Lasioderma minutum Lindberg, 1950 & NS & ES & * & * & + & * & * & - \\
\hline Mezium americanum (Laporte, 1840) & IP & & * & * & + & $*$ & * & - \\
\hline Nicobium villosum (Brullé, 1838) & NS & & * & * & + & * & * & - \\
\hline Piarus basalis Wollaston, 1862 & NS & ES & * & * & + & * & $*$ & - \\
\hline Sphaericus gibbicollis Wollaston, 1862 & NS & ES & * & * & + & * & * & - \\
\hline Stagetus hirtulus ssp. orientalis Israelson, 1974 & NS & ESS & - & * & - & + & - & - \\
\hline Anthicidae & & & & & & & & \\
\hline Aulacoderus canariensis (Wollaston, 1864) & NS & ES & - & * & + & + & - & - \\
\hline Apionidae & & & & & & & & \\
\hline Dieckmanniellus nitidulus Gyllenhal, 1838 & NO & & - & * & - & - & + & - \\
\hline Belidae & & & & & & & & \\
\hline Aglycyderes setifer Westwood, 1863 & NS & ES & - & * & + & - & - & - \\
\hline Cantharidae & & & & & & & & \\
\hline Malthinus depauperatus Wollaston, 1862 & NS & ES & * & * & + & * & $*$ & - \\
\hline Malthinus lindbergi Palm, 1975 & NS & ES & - & * & - & - & + & - \\
\hline
\end{tabular}




\begin{tabular}{|c|c|c|c|c|c|c|c|c|}
\hline Carabidae & & & & & & & & \\
\hline Amara rufescens Dejean, 1829 & NP & & - & * & - & - & + & - \\
\hline $\begin{array}{l}\text { Bembidion atlanticum ssp. atlanticum } \\
\text { Wollaston, } 1854\end{array}$ & NS & & - & * & - & + & - & - \\
\hline Bembidion fortunatum Wollaston, 1871 & NS & ES & - & * & - & + & - & - \\
\hline Calosoma olivieri Dejean, 1831 & NP & & - & * & - & + & + & - \\
\hline Cymindis discophora Chaudoir, 1873 & $\mathrm{NO}$ & & - & * & + & - & + & + \\
\hline Cymindis marginella Brullé, 1838 & NS & ES & - & * & + & + & - & - \\
\hline Cymindis moralesi Mateu, 1979 & NS & ES & - & * & - & - & + & - \\
\hline $\begin{array}{l}\text { Cymindis suturalis ssp. pseudosuturalis } \\
\text { Bedel, } 1906\end{array}$ & NP & & * & * & + & * & * & - \\
\hline Masoreus affinis ssp. arenicola Wollaston, 1863 & IP & & - & * & + & - & + & - \\
\hline Microlestes gomerensis Lindberg, 1953 & NP & & - & * & - & - & + & - \\
\hline Olisthopus elongatus Wollaston, 1854 & NS & & - & * & + & + & - & - \\
\hline Orthomus berytensis Reiche and Saulcy, 1854 & NP & & - & * & - & - & + & - \\
\hline Orthomus discors (Wollaston, 1864) & NS & & - & * & + & + & - & - \\
\hline Orzolina thalassophila Machado, 1987 & NS & EG & * & * & + & * & * & - \\
\hline $\begin{array}{l}\text { Paradromius exornatus ssp. furvus } \\
\text { Machado, } 1992\end{array}$ & NS & ES & - & * & + & - & + & - \\
\hline Philorhizus incertus (Wollaston, 1864) & NS & ES & * & * & + & * & * & - \\
\hline Poecilus wollastoni Wollaston, 1854 & NP & & - & * & - & + & - & - \\
\hline Pogonus chalceus Marsham, 1802 & NO & & * & * & * & * & * & + \\
\hline Scarites buparius Förster, 1771 & NP & & - & * & - & - & + & - \\
\hline Sphodrus leucophthalmus Linnaeus, 1758 & IS & & - & * & - & + & - & - \\
\hline Syntomus fuscomaculatus Motschulsky, 1845 & NO & & - & * & - & - & + & - \\
\hline Syntomus lancerotensis (Wollaston, 1864) & NS & ES & - & * & - & + & - & - \\
\hline Trechus detersus Wollaston, 1864 & NS & ES & - & * & - & + & - & + \\
\hline Chrysomelidae & & & & & & & & \\
\hline Aphthona convexior Lindberg, 1950 & NS & ES & - & * & - & - & + & - \\
\hline $\begin{array}{l}\text { Aphthona wachnitzae (Madar and Madar, } \\
1968 \text { ) }\end{array}$ & NO & & * & * & + & * & * & - \\
\hline $\begin{array}{l}\text { Chrysolina lucidicollis ssp. grossepunctata } \\
\text { Lindberg, } 1950\end{array}$ & NS & ES & - & * & - & - & + & - \\
\hline Cryptocephalus nitidicollis Wollaston, 1864 & NS & & * & * & + & * & * & - \\
\hline Macrocoma divisa (Wollaston, 1864) & NS & ES & - & * & + & - & + & - \\
\hline Macrocoma oromianum Daccordi, 1978 & NS & & - & * & - & + & - & - \\
\hline Oxylepus deflexicollis Boheman, 1862 & NS & & - & * & + & + & - & - \\
\hline Clambidae & & & & & & & & \\
\hline Clambus complicans Wollaston, 1864 & NS & ES & - & $*$ & + & - & - & - \\
\hline
\end{tabular}




\begin{tabular}{|c|c|c|c|c|c|c|c|c|}
\hline \multicolumn{9}{|l|}{ Cleridae } \\
\hline Canariclerus paivae (Wollaston, 1862) & NS & EG & - & * & + & - & - & - \\
\hline Necrobia rufipes De Geer, 1775 & IP & & - & * & - & + & - & - \\
\hline \multicolumn{9}{|l|}{ Coccinelidae } \\
\hline Coccinella algerica Kovar, 1977 & NO & & - & * & + & + & + & - \\
\hline Lindorus lophantae (Blaisdell, 1892) & IP & & * & * & + & * & * & - \\
\hline Nephus flavopictus (Wollaston, 1854) & NP & & * & * & + & * & * & - \\
\hline Parexochomus quadriplagiatus (Wollaston, 1864) & NS & ES & - & $*$ & - & + & - & - \\
\hline Scymnus canariensis Wollaston, 1864 & NS & ES & - & * & + & + & - & - \\
\hline \multicolumn{9}{|l|}{ Curculionidae } \\
\hline Amaurorhinus clermonti Desbrochers, 1908 & NS & & * & * & + & * & * & - \\
\hline Amaurorhinus monizianus Wollaston, 1860 & NS & & + & * & + & + & + & - \\
\hline Aphanarthrum affine Wollaston, 1860 & NS & & - & * & - & - & + & + \\
\hline Aphanarthrum bicinctum Wollaston, 1860 & NS & & - & * & + & - & + & - \\
\hline Aphanarthrum jubae Wollaston, 1860 & NS & ES & * & * & + & * & * & + \\
\hline Canariacalles lanzarotensis (Stüben, 2000) & NS & ES & * & * & + & * & $*$ & - \\
\hline Coniocleonus excoriatus (Gyllenhal, 1834) & NO & & - & * & - & - & + & - \\
\hline Coniocleonus variolosus (Wollaston, 1864) & $\mathrm{NO}$ & & * & * & * & * & * & + \\
\hline Conorhynchus conicirostris (Olivier, 1807) & NO & & - & * & - & + & - & + \\
\hline Depresseremiarhinus brevitarsis (Wollaston, 1864) & NS & ES & - & * & - & + & - & + \\
\hline Gronops fasciatus Küster, 1851 & NO & & * & * & + & * & * & - \\
\hline Herpisticus oculatus Wollaston, 1864 & NS & EG & - & * & - & + & - & - \\
\hline Laparocerus dispar Wollaston, 1864 & NS & ES & * & * & + & * & * & - \\
\hline Laparocerus rasus Wollaston, 1864 & NS & ES & * & $*$ & + & * & $*$ & - \\
\hline Liparthrum bicaudatum Wollaston, 1865 & NS & ES & * & * & + & * & * & - \\
\hline Liparthrum inarmatum Wollaston, 1860 & NP & & * & * & + & * & * & - \\
\hline Mesites fusiformis Wollaston, 1861 & NS & ES & - & * & - & + & + & + \\
\hline Onycholips bifurcatus Wollaston, 1861 & NS & ES & - & * & + & - & + & - \\
\hline Pentatemnus arenarius Wollaston, 1861 & NS & & - & * & + & - & + & + \\
\hline Pselactus caulium (Wollaston, 1861) & NS & ES & - & $*$ & + & + & - & - \\
\hline Rhytideres plicatus Olivierm 1790 & IS & & - & $*$ & - & - & + & - \\
\hline Sibinia sericea Wollaston, 1864 & NS & ES & - & * & - & + & - & - \\
\hline Sitona gressorius Fabricius, 1775 & NO & & - & * & - & - & + & - \\
\hline Smicronyx albosquamosus Wollaston, 1854 & NO & & * & * & + & * & * & - \\
\hline Tychius striatulus Gyllenhal, 1836 & NO & & * & * & * & * & * & + \\
\hline \multicolumn{9}{|l|}{ Dermestidae } \\
\hline Dermestes frischi Kugelann, 1792 & NO & & + & * & - & + & - & - \\
\hline
\end{tabular}




\begin{tabular}{|c|c|c|c|c|c|c|c|c|}
\hline Thorictus vestitus Wollaston, 1864 & NS & ES & * & * & + & * & * & - \\
\hline \multicolumn{9}{|l|}{ Dytiscidae } \\
\hline Herophydrus musicus Klug, 1834 & NS & & - & * & - & + & - & - \\
\hline Hygrotus confluens Fabricius, 1787 & NS & & - & * & - & + & - & - \\
\hline \multicolumn{9}{|l|}{ Endomychidae } \\
\hline Holoparamecus singularis Becker, 1817 & NO & & - & * & - & + & - & - \\
\hline \multicolumn{9}{|l|}{ Gietellidae } \\
\hline $\begin{array}{l}\text { Gietella fortunata Constantin and } \\
\text { Menier, } 1990\end{array}$ & NS & ES & - & * & - & + & - & - \\
\hline \multicolumn{9}{|l|}{ Histeridae } \\
\hline Eutriptus putricola Wollaston, 1862 & NS & & - & * & - & - & + & - \\
\hline Gnathoncus rotundatus Kugelann, 1792 & NO & & - & * & - & - & + & - \\
\hline Hypocaccus mundus Wollaston, 1864 & NS & ES & * & * & * & * & * & + \\
\hline Saprinus chalcites Illiger, 1807 & NP & & - & * & - & - & + & - \\
\hline Saprinus moyses Marseul, 1862 & NP & & * & * & * & * & * & + \\
\hline Saprinus proximus Wollaston, 1865 & NS & ES & - & * & - & + & + & - \\
\hline \multicolumn{9}{|l|}{ Laemophloeidae } \\
\hline Cryptolestes spartii (Curtis, 1834) & NO & & * & * & + & * & * & - \\
\hline \multicolumn{9}{|l|}{ Latridiidae } \\
\hline Corticaria maculosa ssp. lineata Johnson, 1974 & NS & ESS & * & * & + & * & * & + \\
\hline Metophthalmus ferrugineus Wollaston, 1865 & NS & ES & * & * & + & * & * & - \\
\hline \multicolumn{9}{|l|}{ Melyridae } \\
\hline Attalus chrysanthemi Wollaston, 1862 & NS & ES & * & * & + & * & * & - \\
\hline Attalus hariensis Evers, 1960 & NS & ES & * & * & + & $*$ & * & - \\
\hline Attalus laevicollis Wollaston, 1862 & NS & & * & * & + & * & * & - \\
\hline Attalus ochraceus Lindberg, 1950 & NS & ES & - & * & - & + & + & - \\
\hline Dasytes lanzarotensis Palm, 1974 & NS & ES & - & * & + & + & + & - \\
\hline Ifnidius petricola Plata, 1987 & NS & ES & - & * & - & + & - & - \\
\hline \multicolumn{9}{|l|}{ Monotomidae } \\
\hline Europs impressicollis Wollaston, 1854 & NS & & - & * & - & + & - & - \\
\hline \multicolumn{9}{|l|}{ Nitidulidae } \\
\hline Carophilus ligneus Murray, 1864 & $\mathrm{NO}$ & & * & * & + & $*$ & $*$ & - \\
\hline Epuraea ocularis (Fairmaire, 1849) & NO & & $*$ & * & - & $*$ & * & - \\
\hline Meligethes varicollis Wollaston, 1854 & NO & & * & * & * & $*$ & * & + \\
\hline \multicolumn{9}{|l|}{ Oedemeridae } \\
\hline Chitona suturalis (Olivider, 1811) & NP & & * & * & * & $*$ & * & + \\
\hline \multicolumn{9}{|l|}{ Ptinidae } \\
\hline Mezium americanum Laporte, 1840 & IP & & - & * & - & + & - & - \\
\hline
\end{tabular}




\begin{tabular}{|c|c|c|c|c|c|c|c|c|}
\hline \multicolumn{9}{|l|}{ Scarabidae } \\
\hline Pachydema menieri Baraud, 1985 & NS & ES & - & * & - & + & - & - \\
\hline Pachydema wollastoni Peyerimhoff, 1928 & NS & ES & * & * & * & * & * & + \\
\hline \multicolumn{9}{|l|}{ Scraptiidae } \\
\hline Anaspis canariensis Ermisch, 1967 & NS & ES & * & * & + & * & * & - \\
\hline \multicolumn{9}{|l|}{ Staphylinidae } \\
\hline Atheta coriaria (Kraatz, 1856) & NO & & * & * & + & * & * & - \\
\hline Gyrohypnus marginalis (Wollaston, 1862) & NS & ES & * & * & * & * & $*$ & + \\
\hline $\begin{array}{l}\text { Leptobium nigricolle ssp. nigricolle } \\
\text { Wollaston, } 1862\end{array}$ & NS & ESS & - & * & + & + & + & - \\
\hline Leptobium ruficolle (Wollaston, 1862) & NS & ES & * & * & * & * & $*$ & + \\
\hline Sunius brevipennis (Wollaston, 1864) & NS & ES & * & * & + & * & * & - \\
\hline \multicolumn{9}{|l|}{ Tenebrionidae } \\
\hline Arthrodeis costifrons Wollaston, 1864 & NS & ES & - & * & + & - & + & + \\
\hline Arthrodeis geotrupoides Wollaston, 1864 & NS & ES & * & * & * & * & $*$ & + \\
\hline Arthrodeis hartungii Wollaston, 1864 & NS & ES & - & * & + & + & - & - \\
\hline Arthrodeis inflatus Wollaston, 1864 & NS & ES & - & * & + & - & + & - \\
\hline Arthrodeis laticollis (Brullé, 1838) & NS & ES & - & * & - & - & + & + \\
\hline Arthrodeis malleatus Wollaston, 1864 & NS & ES & + & * & + & - & - & - \\
\hline Arthrodeis punctatulus Wollaston, 1864 & NS & ES & + & * & - & + & + & + \\
\hline Arthrodeis subciliatus Wollaston, 1864 & NS & ES & * & * & $*$ & * & * & + \\
\hline Blaps alternans Brullé, 1838 & IP & & - & * & - & + & + & - \\
\hline Blaps gigas Linnaeus, 1767 & IP & & - & * & - & - & + & - \\
\hline Boromorphus parvus Wollaston, 1864 & NS & ES & - & * & + & - & - & - \\
\hline Clitobius ovatus (Erichson, 1843) & NP & & * & * & * & * & * & + \\
\hline Gonocephalum affine Billberg, 1815 & $\mathrm{NO}$ & & - & * & - & - & + & - \\
\hline Gonocephalum oblitum (Wollaston, 1864) & NS & ES & - & * & + & + & + & + \\
\hline Gonocephalum patruele Erichson, 1843 & NP & & - & * & - & + & - & + \\
\hline Hegeter amaroides Solier, 1835 & NS & ES & + & * & + & + & + & - \\
\hline Hegeter deyrollei (Wollaston, 1864) & NS & ES & * & * & + & * & * & + \\
\hline Hegeter tristis Fabricius, 1792 & NP & & - & * & + & + & + & - \\
\hline Melanochrus lacordairi Wollaston, 1864 & NS & $\mathrm{EG}$ & - & * & - & - & + & + \\
\hline Melasmana lineatum (Brullé, 1838) & NS & EG & - & * & + & + & + & + \\
\hline Nesotes aethiops (Wollaston, 1864) & NS & ES & * & * & + & * & * & + \\
\hline Nesotes picescens (Wollaston, 1864) & NS & ES & * & * & + & * & * & + \\
\hline Nesotes porrectus (Wollaston, 1864) & NS & ES & * & * & + & * & * & - \\
\hline Nesotes sabulicola Israelson, 1980 & NS & ES & $*$ & * & + & * & * & - \\
\hline Oxycarops fuscipes_(Brullé, 1838) & NS & $\mathrm{EG}$ & - & $*$ & + & - & + & - \\
\hline
\end{tabular}




\begin{tabular}{|c|c|c|c|c|c|c|c|c|}
\hline Paivaea hispida (Brullé, 1838) & NS & ES & + & * & + & + & - & + \\
\hline Palorus euphorbiae (Wollaston, 1862) & NS & ES & - & * & - & + & - & - \\
\hline Phaleria cadaverina (Fabricius, 1792) & NS & & * & * & * & * & * & + \\
\hline Phaleria ornata Wollaston, 1864 & NS & ES & - & * & - & - & + & - \\
\hline Phtora angusta (Wollaston, 1861) & NS & & * & * & * & * & * & + \\
\hline Pimelia lutaria Brullé, 1838 & NS & ES & - & * & - & - & + & - \\
\hline Pseudoseriscius fonti (Escalera, 1923) & NS & & - & * & + & - & + & - \\
\hline Zophosis bicarinata ssp. plicata Brullé, 1838 & NS & ES & - & * & + & + & + & + \\
\hline \multicolumn{9}{|l|}{ ORDER LEPIDOPTERA } \\
\hline \multicolumn{9}{|l|}{ Arctiidae } \\
\hline Utetheisa pulchella Linnaeus, 1758 & $\mathrm{NO}$ & & - & * & - & + & - & - \\
\hline \multicolumn{9}{|l|}{ Blastobasidae } \\
\hline Blastobasis phycidella (Zeller, 1839) & NO & & - & - & + & - & - & - \\
\hline \multicolumn{9}{|l|}{ Geometridae } \\
\hline Aspitates collinaria Holt-White, 1894 & NS & ES & - & * & - & + & - & - \\
\hline Gymnoscelis insulariata Stainton, 1859 & NS & & - & * & - & + & - & - \\
\hline Microloxia schmitzi Hausmann, 1994 & NP & & - & * & - & - & + & - \\
\hline Scopula guancharia Alpheraky, 1889 & NS & & - & * & - & + & + & - \\
\hline \multicolumn{9}{|l|}{ Gracillariidae } \\
\hline Acrocercops hedemanni (Rebel, 1896) & NS & & - & - & + & - & - & - \\
\hline \multicolumn{9}{|l|}{ Noctuidae } \\
\hline Agrotis ipsilon (Hufnagel, 1766) & $\mathrm{NO}$ & & - & - & + & - & - & - \\
\hline Agrotis lanzarotensis Rebel, 1894 & NS & ES & - & - & + & - & - & - \\
\hline Cardepia deserticola Hampson, 1905 & NP & & - & * & - & - & + & - \\
\hline Chrysodeixis chalcites Esper, [1789] & IP & & - & * & - & + & - & - \\
\hline $\begin{array}{l}\text { Eremobastis loslobensis Fischer, Saldaitis and } \\
\text { Ivinskis, } 2007\end{array}$ & NS & ES & $*$ & * & $*$ & $*$ & * & + \\
\hline Euxoa canariensis Rebel, 1902 & NS & & - & * & - & - & + & - \\
\hline $\begin{array}{l}\text { Heliothis peltigera Denis and Schiffermüller, } \\
1775\end{array}$ & NO & & - & * & - & + & - & - \\
\hline Mniotype usurpatrix ssp. hariana Pinker, 1868 & NS & ES & - & * & - & + & - & - \\
\hline Cryphia simonyi (Rogenhofer, 1889) & NS & ES & - & * & - & + & - & - \\
\hline Paradrina lanzarotensis (Pinker, 1962) & NS & ES & - & * & - & - & + & - \\
\hline Spodoptera exigua Hübner, 1808 & NP & & - & * & - & - & + & - \\
\hline Trichoplusia ni Hübner, [1803] & IP & & - & * & - & - & + & - \\
\hline \multicolumn{9}{|l|}{ Nymphalidae } \\
\hline Vanessa cardui Linnaeus, 1758 & NP & & - & * & + & + & + & - \\
\hline
\end{tabular}




\begin{tabular}{|c|c|c|c|c|c|c|c|c|}
\hline \multicolumn{9}{|l|}{ Pieridae } \\
\hline Colias crocea Geoffroy, 1785 & NS & & - & * & + & + & + & - \\
\hline \multicolumn{9}{|l|}{ Pyralidae } \\
\hline Euchromius ocelleus (Haworth, 1811) & $\mathrm{NO}$ & & - & - & + & - & - & - \\
\hline $\begin{array}{l}\text { Nomophila noctuella (Denis and Schiffermüller, } \\
1775 \text { ) }\end{array}$ & IP & & - & - & + & - & - & - \\
\hline \multicolumn{9}{|l|}{ Sphingidae } \\
\hline Hippotion celerio Linnaeus, 1758 & NP & & - & * & - & - & + & - \\
\hline Hyles livornica ssp. livornica Esper, 1779 & NP & & - & * & - & - & + & - \\
\hline Hyles tithymali ssp. tithymali Boisduval, 1832 & NP & & - & * & - & - & + & + \\
\hline Macroglossum stellatarum (Linnaeus, 1758) & NP & & - & - & + & - & - & - \\
\hline \multicolumn{9}{|l|}{ Tineidae } \\
\hline $\begin{array}{l}\text { Trichophaga robinsoni Gaedike and } \\
\text { Karsholt, } 2001\end{array}$ & NO & & - & - & + & - & - & - \\
\hline \multicolumn{9}{|l|}{ Tortricidae } \\
\hline Acrolita subsequana Herrich-Schäffer, 1851 & NS & & - & - & + & - & - & - \\
\hline \multicolumn{9}{|l|}{ ORDER DIPTERA } \\
\hline \multicolumn{9}{|l|}{ Acroceridae } \\
\hline Acrocera cabrerae Frey, 1936 & NS & ES & - & - & + & - & - & - \\
\hline \multicolumn{9}{|l|}{ Anthomyiidae } \\
\hline Anthomyia xanthopus (Hennig, 1974) & NP & & * & * & * & * & * & + \\
\hline \multicolumn{9}{|l|}{ Asilidae } \\
\hline Habropogon pertusus Becker, 1908 & NS & ES & - & * & - & + & - & - \\
\hline Promachus consanguineus (Macquart, 1839) & NS & ES & * & * & * & * & * & + \\
\hline Saropogon punctipennis Frey, 1958 & NS & ES & - & * & + & + & - & - \\
\hline \multicolumn{9}{|l|}{ Bombyliidae } \\
\hline Anastoechus latifrons (Macquart, 1839) & NS & & * & * & $*$ & * & * & + \\
\hline Dischistus atlanticus (Santos Abreu, 1926) & NS & ES & - & * & - & - & + & + \\
\hline \multicolumn{9}{|l|}{ Calliphoridae } \\
\hline Calliphora vicina Robineau-Desvoidy, 1830 & IP & & - & - & + & - & - & - \\
\hline Lucilia sericata Meigen, 1826 & II & & + & * & - & + & - & + \\
\hline \multicolumn{9}{|l|}{ Coelopidae } \\
\hline Malacomyia sciomyzina (Haliday, 1833) & NO & & - & - & + & - & - & - \\
\hline \multicolumn{9}{|l|}{ Hippoboscidae } \\
\hline Ornithophila metallica (Shiner, 1864) & NS & & - & - & + & - & - & - \\
\hline \multicolumn{9}{|l|}{ Limoniidae } \\
\hline Geranomyia canariensis Bergroth, 1889 & NS & & - & * & - & + & - & - \\
\hline
\end{tabular}




\begin{tabular}{|c|c|c|c|c|c|c|c|c|}
\hline \multicolumn{9}{|l|}{ Stratiomydae } \\
\hline Alliophleps elliptica Becker, 1908 & NS & EG & - & - & + & - & - & - \\
\hline \multicolumn{9}{|l|}{ Syrphidae } \\
\hline Episyrphus balteatus De Geer, 1776 & NS & & - & * & - & + & + & - \\
\hline Eupeodes corollae (Fabricius, 1794) & NP & & * & * & * & * & * & + \\
\hline Paragus tibialis Fallén, 1817 & NS & & - & * & - & + & - & - \\
\hline Syritta pipiens Linnaeus, 1758 & NS & & - & * & - & - & + & - \\
\hline \multicolumn{9}{|l|}{ Tachinidae } \\
\hline Mintho compressa Fabricius, 1787 & NS & & - & * & - & + & - & - \\
\hline \multicolumn{9}{|l|}{ Tephritidae } \\
\hline Trupanea amoena (Frauenfeld, 1857) & NO & & - & - & + & - & - & - \\
\hline \multicolumn{9}{|l|}{ Tethinidae } \\
\hline Tethina grossipes (Becker, 1908) & NS & & * & * & $*$ & * & $*$ & + \\
\hline Tethina marmorata (Becker, 1908) & NS & ES & + & * & - & - & - & - \\
\hline \multicolumn{9}{|l|}{ Therevidae } \\
\hline Irwiniella purpurariae Frey, 1958 & NS & ES & - & * & - & + & - & - \\
\hline \multicolumn{9}{|l|}{ Trixosceliidae } \\
\hline Trixoscelis puncticornis Becker, 1907 & NP & & + & * & - & - & - & - \\
\hline \multicolumn{9}{|l|}{ Vermileonidae } \\
\hline Lampromyia hemmingseni Stuckenberg, 1971 & NS & ES & - & - & + & - & - & - \\
\hline \multicolumn{9}{|l|}{ ORDER HYMENOPTERA } \\
\hline \multicolumn{9}{|l|}{ Anthoporidae } \\
\hline Amegilla quadrifasciata (Villers, 1790) & NP & & - & * & - & + & + & - \\
\hline $\begin{array}{l}\text { Anthophora alluaudi ssp. fuerteventurae } \\
\text { Lieftinck, } 1958\end{array}$ & NS & ESS & - & * & - & + & - & + \\
\hline Tetralonia maroccana Dusmet, 1928 & NP & & * & $*$ & $*$ & $*$ & * & + \\
\hline Thyreus histrionicus (Illiger, 1806) & NP & & - & - & + & - & - & - \\
\hline \multicolumn{9}{|l|}{ Chrysididae } \\
\hline Chrysis hohmanni Linsenmaier, 1993 & NS & ES & - & * & - & - & + & - \\
\hline \multicolumn{9}{|l|}{ Crabronidae } \\
\hline Andrena rutila ssp. xanthoscelis Brullé, 1839 & NS & ESS & - & $*$ & - & - & + & - \\
\hline Crossocerus lindbergi (Beaumont, 1954) & NS & ES & * & * & $*$ & $*$ & * & + \\
\hline Dryudella sepulchralis (Beaumont, 1968) & NS & ES & * & * & * & * & * & + \\
\hline Harpactus guichardi (Beaumont, 1968) & NS & ES & * & * & * & $*$ & * & + \\
\hline Lasioglossum loetum (Brullé, 1839) & NS & ES & - & * & - & + & - & + \\
\hline Lasioglossum morio ssp. cordiale Pérez, 1903 & NP & & - & * & - & + & - & - \\
\hline Lasioglossum phoenicurum (Warncke, 1975) & NP & & * & * & $*$ & * & * & + \\
\hline Lindenius efferenus (Kohl, 1915) & NP & & * & * & $*$ & * & * & + \\
\hline
\end{tabular}




\begin{tabular}{|c|c|c|c|c|c|c|c|c|}
\hline Liris micans (Spinola, 1806) & NP & & - & - & + & - & - & + \\
\hline Miscophus guichardi Beaumont, 1968 & NS & ES & - & $*$ & - & + & + & - \\
\hline Miscophus helveticus Kohl, 1883 & NP & & - & * & - & - & + & + \\
\hline Miscophus mucronatus (Fabricius, 1793) & $\mathrm{NP}$ & & * & * & * & $*$ & $*$ & + \\
\hline Nomioides deceptor Saunders, 1937 & NS & & - & * & - & + & + & - \\
\hline Oxybelus cocacolae Verhoeff, 1968 & NP & & - & $*$ & - & - & + & - \\
\hline Panurgus dentipes Latreille, 1811 & NS & & * & * & * & * & * & + \\
\hline Solierella canariensis Saunders, 1904 & NS & ES & * & $*$ & $*$ & $*$ & $*$ & + \\
\hline \multicolumn{9}{|l|}{ Eumenidae } \\
\hline Ancistrocerus kerneri (Dalla Torre, 1904) & NS & ES & - & * & - & + & - & - \\
\hline Leptochilus fortunatus Blüthgen, 1958 & NS & ES & - & * & + & - & + & + \\
\hline Leptochilus replenus Giordani-Soika, 1974 & NS & ES & $*$ & * & * & * & $*$ & + \\
\hline \multicolumn{9}{|l|}{ Formicidae } \\
\hline Camponotus feai Emery, 1882 & NS & ES & - & * & + & + & + & - \\
\hline Camponotus carinatus (Brullé, 1839) & NS & ES & - & - & + & - & - & - \\
\hline Cardiocondyla emeryi Forel, 1839 & II & & - & * & - & + & - & - \\
\hline Crematogaster alluaudi Emery, 1893 & NS & ES & - & * & - & + & - & - \\
\hline $\begin{array}{l}\text { Crematogaster laestrygon ssp. canariensis } \\
\text { Emery, } 1926\end{array}$ & NS & ESS & - & * & - & - & + & - \\
\hline $\begin{array}{l}\text { Leptothorax rottenbergi ssp. scabriosus } \\
\text { Santschi, } 1919\end{array}$ & $\mathrm{NO}$ & & - & * & - & + & - & - \\
\hline Messor hesperius Santschi, 1927 & $\mathrm{NO}$ & & - & * & + & + & + & - \\
\hline Monomorium hesperium Emery, 1895 & NS & ES & - & * & - & - & + & - \\
\hline $\begin{array}{l}\text { Plagiolepis barbara ssp. canariensis } \\
\text { Santschi, } 1920\end{array}$ & NS & ESS & - & * & + & + & + & - \\
\hline Tetramorium depressum Forel, 1892 & $\mathrm{NO}$ & & + & * & + & + & - & - \\
\hline \multicolumn{9}{|l|}{ Ichneumonidae } \\
\hline $\begin{array}{l}\text { Cryptus praefortis ssp. insularis } \\
\text { Van Rossem, } 1989\end{array}$ & NS & ESS & * & - & + & - & - & - \\
\hline \multicolumn{9}{|l|}{ Masaridae } \\
\hline Quartinia canariensis Blüthgen, 1958 & NS & ES & * & * & * & * & $*$ & + \\
\hline \multicolumn{9}{|l|}{ Megrachilidae } \\
\hline Chalicodoma sicula ssp. balearica Tkalcu, 1977 & NP & & - & * & + & - & - & - \\
\hline Megachile binominata Smith, 1853 & NS & ES & $*$ & * & $*$ & $*$ & $*$ & + \\
\hline \multicolumn{9}{|l|}{ Pompolidae } \\
\hline Agenioideus oasis Haupt, 1962 & NP & & - & * & - & - & + & - \\
\hline Pareiocurgus violaceipennis (Brullé, 1839) & NP & & * & * & * & $*$ & $*$ & + \\
\hline Tachyagetes aemulans (Haupt, 1928) & NS & & - & - & + & - & - & - \\
\hline
\end{tabular}




\begin{tabular}{|c|c|c|c|c|c|c|c|c|}
\hline Tachyagetes lanzarotus Wolf, 1993 & NS & ES & - & * & - & + & + & - \\
\hline \multicolumn{9}{|l|}{ Pteromalidae } \\
\hline Heocolax formiciformis Westwood, 1832 & NP & & * & - & + & - & - & - \\
\hline \multicolumn{9}{|l|}{ Scoliidae } \\
\hline Micromeriella aureola ssp. elegans Brullé, 1839 & NS & ESS & - & * & - & - & + & - \\
\hline \multicolumn{9}{|l|}{ Sphecidae } \\
\hline Podalonia tydei Le Guillou, 1841 & NP & & - & * & - & - & + & - \\
\hline \multicolumn{9}{|l|}{ Tiphiidae } \\
\hline Poecilotiphia gracilis Brullé, 1839 & NS & ES & - & * & - & + & - & - \\
\hline \multicolumn{9}{|l|}{ ORDER PSEUDOESCORPIONS } \\
\hline \multicolumn{9}{|l|}{ Cheliferidae } \\
\hline Canarichelifer teneriffae Beier, 1965 & NS & EG & * & - & + & - & - & - \\
\hline \multicolumn{9}{|l|}{ Chtoniidae } \\
\hline Paraliochthonius canariensis Vachon, 1961 & NS & ES & * & - & + & - & - & - \\
\hline \multicolumn{9}{|l|}{ Garypidae } \\
\hline Garypus beauvoisi Audouin, 1826 & NP & & * & - & + & - & - & + \\
\hline \multicolumn{9}{|l|}{ Geogarypidae } \\
\hline Geogarypus minor L. Koch, 1873 & NP & & * & - & + & - & - & - \\
\hline \multicolumn{9}{|l|}{ Olpiidae } \\
\hline Calocheirus canariensis (Beier, 1970) & NS & ES & * & - & + & - & - & - \\
\hline Olpium canariense Beier, 1965 & NS & ES & * & - & + & - & - & - \\
\hline \multicolumn{9}{|l|}{ ORDER SIPHONAPTERA } \\
\hline \multicolumn{9}{|l|}{ Pulicidae } \\
\hline $\begin{array}{l}\text { Xenopsylla gratiosa Jordan and Rothschild, } \\
1923\end{array}$ & IS & & * & - & + & - & - & - \\
\hline \multicolumn{9}{|l|}{ ORDER THYSANOPTERA } \\
\hline \multicolumn{9}{|l|}{ Aeolothripidae } \\
\hline Melanthrips areolatus Priesner, 1936 & NO & & * & * & * & * & * & + \\
\hline Rhipidothrips unicolor zur Strassen, 1965 & $\mathrm{NO}$ & & * & * & * & * & * & + \\
\hline \multicolumn{9}{|l|}{ Phlaeothripidae } \\
\hline Haplothrips balsaminus zur Strassen, 1966 & NO & & * & * & * & * & * & + \\
\hline Haplothrips guanche zur Strassen, 1966 & NO & & * & * & * & * & * & + \\
\hline \multicolumn{9}{|l|}{ Thripidae } \\
\hline Odontothrips karnyi Priesner, 1924 & NO & & $*$ & * & * & $*$ & * & + \\
\hline
\end{tabular}




\begin{tabular}{|c|c|c|c|c|c|c|c|c|}
\hline Phylum Mollusca & Or & En & RE & $\mathrm{RO}$ & MC & AL & LG & LO \\
\hline \multicolumn{9}{|l|}{ ORDER PULMonata } \\
\hline \multicolumn{9}{|l|}{ Enidae } \\
\hline Napaeus huttereri Henríquez, 1991 & NS & EG & - & $*$ & - & + & - & * \\
\hline \multicolumn{9}{|l|}{ Ferussaciidae } \\
\hline Ferussacia vitrea (Webb and Berthelot, 1833) & NS & ES & - & * & + & - & - & * \\
\hline \multicolumn{9}{|l|}{ Helicidae } \\
\hline Hemicycla flavistoma Ibáñez and Alonso, 1991 & NS & ES & - & * & - & + & - & * \\
\hline $\begin{array}{l}\text { Hemicycla sarcostoma (Webb and } \\
\text { Berthelot, 1833) }\end{array}$ & NS & ES & - & $*$ & + & + & - & * \\
\hline Otala lactea O. F. Müller, 1774 & IP & & - & * & + & - & - & * \\
\hline Theba geminata (Mousson, 1857) & NS & ES & + & * & + & + & + & + \\
\hline \multicolumn{9}{|l|}{ Parmacellidae } \\
\hline Cryptella alegranzae Hutterer and Groh, 1991 & NS & IE & - & * & - & + & - & * \\
\hline \multicolumn{9}{|l|}{ Subulinidae } \\
\hline Rumina decollata Linnaeus, 1758 & NP & & - & * & + & - & - & + \\
\hline \multicolumn{9}{|l|}{ Trissexodontidae } \\
\hline Caracollina lenticula Michaud, 1831 & NP & & - & * & + & - & - & * \\
\hline \multicolumn{9}{|l|}{ Hygromiidae } \\
\hline Canariella plutonia (R.T Lowe, 1861) & NS & EG & - & - & - & - & - & + \\
\hline
\end{tabular}

